Review

\title{
Turbulent collision of inertial particles: Point-particle based, hybrid simulations and beyond
}

\author{
Lian-Ping Wang ${ }^{\mathrm{a}, *}$, Bogdan Rosa ${ }^{\mathrm{a}}$, Hui Gao ${ }^{\mathrm{a}}$, Guowei He ${ }^{\mathrm{b}}$, Guodong Jin ${ }^{\mathrm{b}}$ \\ a Department of Mechanical Engineering, University of Delaware, 126 Spencer Laboratory, Newark, DE 19716-3140, USA \\ ${ }^{\mathrm{b}}$ The State Key Laboratory of Nonlinear Mechanics, Institute of Mechanics, Chinese Academy of Sciences, Beijing 100080, PR China
}

\section{A R T I C L E I N F O}

\section{Article history:}

Received 2 June 2008

Received in revised form 10 February 2009

Accepted 10 February 2009

Available online 26 February 2009

\section{Keywords:}

Inertial particles

Droplets

Collision-coalescence

Direct numerical simulation

Large-eddy simulation

Collision kernel

Hydrodynamic interaction

\begin{abstract}
A B S T R A C T
Point-particle based direct numerical simulation (PPDNS) has been a productive research tool for studying both single-particle and particle-pair statistics of inertial particles suspended in a turbulent carrier flow. Here we focus on its use in addressing particle-pair statistics relevant to the quantification of turbulent collision rate of inertial particles. PPDNS is particularly useful as the interaction of particles with small-scale (dissipative) turbulent motion of the carrier flow is mostly relevant. Furthermore, since the particle size may be much smaller than the Kolmogorov length of the background fluid turbulence, a large number of particles are needed to accumulate meaningful pair statistics. Starting from the relative simple Lagrangian tracking of so-called ghost particles, PPDNS has significantly advanced our theoretical understanding of the kinematic formulation of the turbulent geometric collision kernel by providing essential data on dynamic collision kernel, radial relative velocity, and radial distribution function. A recent extension of PPDNS is a hybrid direct numerical simulation (HDNS) approach in which the effect of local hydrodynamic interactions of particles is considered, allowing quantitative assessment of the enhancement of collision efficiency by fluid turbulence. Limitations and open issues in PPDNS and HDNS are discussed. Finally, on-going studies of turbulent collision of inertial particles using large-eddy simulations and particle-resolved simulations are briefly discussed.
\end{abstract}

(c) 2009 Elsevier Ltd. All rights reserved.

\section{Introduction}

Turbulent coagulation, the process of collision-induced merging of particles in a suspension of solid particles or liquid droplets, plays an important role in many natural and industrial processes. Early studies on this subject were motivated by the need to understand the growth of liquid droplets in turbulent clouds (Saffman and Turner, 1956; Almeida, 1976; Pruppacher and Klett, 1997). Subsequent studies, especially addressing the effects of finite particle inertia, were directed to better model and control particle growth in industrial processes, such as droplet growth in wet steam generators (Williams and Crane, 1979) and spray atomization process (O'Rourke and Bracco, 1980), dust separation in cyclones (Abrahamson, 1975), and $\mathrm{TiO}_{2}$ production (Xiong and Pratsinis, 1991). Coagulation of fuel droplets may lead to reduced evaporation and burning rates, resulting in incomplete combustion. Turbulent coagulation can also affect pollutant formation and control (Flagan and Seinfeld, 1988). Smoke ageing (i.e. the increase of particle size in smoke) is a result of particle coagulation, which alters the dispersion and transport of turbulent flames, reduces the rate of smoke particle burn-out, and increases particulate emission (Delichatsios, 1980). A related application is the plankton contact rate which can be affected by small-scale turbulent ocean water flow and is crucial to plankton ecology (Lewis and Pedley, 2000; Schmitt and Seuront, 2008).

Quite a few recent studies of turbulent collision-coalescence were once again driven by cloud microphysics (Pinsky and Khain, 2004; Franklin et al., 2005, 2007; Wang et al., 2005a, 2008; Pinsky et al., 2006, 2007; Ayala et al., 2008a,b). For this particular application, the average particle volume fraction is on the order of $10^{-6}$ (or mass loading on the order of $\left.10^{-3}\right)$, and size of particles $(5-100 \mu \mathrm{m})$ is typically one to two orders of magnitude smaller than the Kolmogorov length $(\sim 1 \mathrm{~mm})$ of the air turbulence. In this paper, we shall focus our attention on such a dilute system, although many of the issues outlined in this paper are relevant to other turbulent particle-laden systems. Since collision-coalescence of particles is of concern and since the inertial response time of particles overlaps with the inter-particle interaction time (say, defined as 10 times the

\footnotetext{
* Corresponding author. Tel.: +1 302831 8160; fax: +1 3028313619 .

E-mail address: lwang@me.udel.edu (L.-P. Wang).
} 
ratio of the geometric collision radius to the differential sedimentation velocity), ${ }^{1}$ the local particle-particle hydrodynamic interaction can prevent some particle pairs from colliding or collision efficiency may be much less than one for cloud droplets of radii less than $50 \mu \mathrm{m}$. While the flow modulation of the background air turbulence by droplets may be neglected, a unique three-way coupling system (Wang et al., 2005a; Ayala et al., 2007), however, must be considered: (a) the carrier-flow turbulence affects the motion of the particles through the interfacial drag; (b) the motion of each particle can be affected by the presence of other particles in the system, either through the strong local near-field binary hydrodynamic interaction or by the cumulative many-body, long-range interactions; and (c) the background carrier-fluid turbulence can also affect the hydrodynamic interactions as the turbulence defines both the far-field conditions and the local environment for the hydrodynamic interactions. In this paper, the terms particles and droplets are used interchangeably, so are the terms hydrodynamic interaction and aerodynamic interaction. In the context of cloud microphysics application, the background air turbulence is relatively weak and therefore the gravitational settling dominates the motion of cloud droplets.

Air turbulence could affect collision-coalescence of cloud droplets in several ways. First, local shear and air acceleration introduce relative fluctuating motion of droplets, leading to larger relative velocity compared to the differential sedimentation (Saffman and Turner, 1956). Second, turbulence-induced preferential concentration of droplets may significantly enhance the average collision rate as local collision rates are proportional to the second moment of local droplet concentrations. The effect of preferential concentration amounts to an enhancement factor (Sundaram and Collins, 1997; Wang et al., 2000, 2005a) known as the radial distribution function (RDF), evaluated at the geometric-collision separation distance $R \equiv a_{1}+a_{2}$ where $a_{1}$ and $a_{2}$ are the radii of two colliding droplets. Third, sedimenting particles bias their trajectories towards regions of downward fluid motion around vortices and could settle significantly faster than the terminal velocity (Maxey, 1987; Wang and Maxey, 1993; Davila and Hunt, 2001), leading to enhanced relative motion between droplets of certain sizes. Finally, air turbulence have been shown to enhance the collision efficiency (Pinsky et al., 1999; Wang et al., 2005a, 2008).

During the last 10 years, we have developed and applied pointparticle based direct simulation approaches to study these effects of air turbulence on collision rate of cloud droplets. The purpose of this paper is to provide a review of these approaches and their contributions to the quantitative understanding of collision statistics of inertial particles, along with some discussion of other related studies. Specifically, we will use the term point-particle based direct numerical simulation (PPDNS) to denote methods where particleparticle local hydrodynamic interactions are neglected. Most of the reported studies of turbulent collisions fall within this category. To incorporate particle-particle local hydrodynamic interactions, we have recently developed a hybrid direct numerical simulation (HDNS) approach (Ayala et al., 2007) in which each droplet introduces a local Stokes disturbance flow. The ensemble of the disturbance flows are then superimposed onto the background air turbulence, as an approximate representation of the three-way coupled turbulent suspension of colliding droplets.

\footnotetext{
${ }^{1}$ For example, consider collisional interaction of $10 \mu \mathrm{m}$ and $30 \mu \mathrm{m}$ water droplets in air, the Stokes inertial response times are $1.3 \mathrm{~ms}$ and $11.8 \mathrm{~ms}$, respectively. The aerodynamic interaction time, estimated using $10\left(a_{1}+a_{2}\right) /\left|v_{p 1}-v_{p 2}\right|$, is about $4 \mathrm{~ms}$, where $a_{1}$ and $a_{2}$ are droplet radii and $v_{p 1}$ and $v_{p 2}$ are terminal velocities. In this case, the smaller droplet has sufficient time to respond to the disturbance flow due to the larger droplet and therefore the collision efficiency is much less than one. For large solid particles, the inertial response time could be much larger than the collision interaction time that is governed by large-scale energetic fluid motion, in that case the hydrodynamic interaction is not so relevant and the collision efficiency is close to one.
}

It should be recognized that the general problem of turbulent coagulation of inertial particles is a complex turbulent multiphase flow problem. A complete description of all the aspects requires a full knowledge of air turbulent motion and dynamics of dropletfluid interactions and droplet-droplet interactions, all of which are three-dimensional and time-dependent in nature. Theoretically, the difficulties in modeling suspension flow of inertial particles include the nonlinear and unsteady nature of the governing equations, the inability to superimpose solutions in general, and multiscale interactions (Koch and Hill, 2001). Experimentally, collision events in a dilute system are rare events occurring at very small scales. It has only recently become feasible to quantitatively measure collision-related pair statistics of inertial particles in a turbulent flow. Wood et al. (2005) measured locations of particles in two space dimensions which provided a two-dimensional projection of RDF. Duru et al. (2007) performed measurement of the growth of aerosol droplets due to coalescence in a turbulent flow chamber, however, such measurement does not allow explicit reduction of pair statistics. Using digital holographic imaging technique, Salazar et al. (2008) measured directly 3D RDF at separation distance on the order of Kolmogorov length and compared their measured data with PPDNS results, showing that quantitative agreement could be obtained if the resolution limitations of the CCD camera in the experiment was properly taken into account in PPDNS. Such holographic imaging system requires computationally intensive postprocessing, and here PPDNS was in fact used to guide the proper interpretation of the measured RDF.

Recently, the potential of using large-eddy simulation approach to study turbulent collision of inertial particles has been discussed (Fede and Simonin, 2006). This is motivated by the fact that LES could be used to address large flow Reynolds numbers. Since pair statistics such as RDF could be governed by small-scale turbulence, it is currently unclear whether LES is an appropriate approach. We will discuss essential difficulties in the LES context.

The paper is organized in terms of the nature of computational approach. We first discuss the methodology and major contributions related to PPDNS. Then the HDNS approach is introduced and discussed. Enough details and the literature survey on PPDNS and HDNS are provided as tutorial for new researchers to the field of turbulent collision. Open issues as well as potential generalization of these approaches are illustrated whenever relevant. Large-eddy simulations and particle-resolved simulations are also briefly discussed, as extensions to PPDNS and HDNS.

\section{Point-particle based DNS}

\subsection{Background air turbulence}

In PPDNS, a direct numerical simulation of homogeneous isotropic turbulence was used to represent the undisturbed or background air flow. The incompressible Navier-Stokes and the continuity equation:

$\frac{\partial \mathbf{U}}{\partial t}=\mathbf{U} \times \omega-\nabla\left(\frac{P}{\rho}+\frac{1}{2} \mathbf{U}^{2}\right)+v \nabla^{2} \mathbf{U}+\mathbf{f}(x, t)$,

$\nabla \cdot \mathbf{U}=0$

were solved in a periodic cubic box using a pseudo-spectral method. $\omega$ and $P$ denote the fluid vorticity and pressure. The term $\mathbf{f}(x, t)$ is a stochastic body force term, restricted to very low wave numbers, that provides an energy source to sustain the air turbulence. Stationary turbulence can be reached since energy propagates from low to high wave numbers; hence, the forcing energy propagates until viscous dissipation becomes active, establishing a quasi-stea- 
dy energy balance. The time evolution was computed using a second-order Adams-Bashforth scheme for the nonlinear terms and a second-order Crank-Nicolson scheme for the viscous term. The pressure was eliminated through the continuity equation.

The small-scale features of the flow are characterized by the Kolmogorov scales defined based on the viscous dissipation rate $\epsilon$ and fluid kinematic viscosity $v$; namely, the Kolmogorov length $\eta$, time $\tau_{k}$, and velocity $v_{k}$ are, respectively,

$\eta=\left(v^{3} / \epsilon\right)^{1 / 4} ; \quad \tau_{k}=(v / \epsilon)^{1 / 2} ; \quad v_{k}=(v \epsilon)^{1 / 4}$

The large-scale features may be characterized by the r.m.s. fluctuation velocity or flow Taylor-microscale Reynolds number

$u^{\prime} \equiv \sqrt{\frac{\langle\mathbf{U} \cdot \mathbf{U}\rangle}{3}}, \quad R_{\lambda}=\sqrt{15}\left(\frac{u^{\prime}}{v_{k}}\right)^{2}$.

The flow domain is discretized uniformly into $N^{3}$ grid points. This grid resolution determines the scale separation, and hence the Reynolds number of the resulting flow. The exact value of flow Reynolds number depends on the resolution parameter $k_{\max } \eta$, the dealiasing scheme, and the forcing method, where $k_{\max }$ is the highest wavenumber realized. Fig. 1 displays the Taylor microscale Reynolds number realized in a few published studies on inertial particles, as a function of $N$. Also shown are data from the stateof-the-art single-phase turbulence simulations by Ishihara et al. (2007) and Donzis et al. (2008). Since the ratio of large-eddy length to Kolmogorov length is proportional to $R^{3 / 2}$, we expect that $R_{\lambda} \propto N^{2 / 3}$. The line in Fig. 1 denotes $R_{\lambda}=3 N^{2 / 3}$. The range of flow Reynolds numbers explored in single-phase turbulent flow simulations covers roughly two orders of magnitude. PPDNS currently resides roughly in the lower half of this range due to the additional computational needs for the particulate phase.

The relatively small $R_{\lambda}$ range and the limited scales in PPDNS are a concern since this implies that not all scales of motion affecting a droplet are resolved in DNS. This currently limits the application of PPDNS to cloud droplets less than $60 \mu \mathrm{m}$ in radius (Ayala et al., 2008a). The flow Reynolds numbers in PPDNS are roughly two orders of magnitude smaller than those in real clouds (Shaw, 2003). It has been argued that since the collision of cloud droplets is governed by two-particle or pair statistics which, for small particles, is mainly determined by the dissipation-range fluid motion, the value of $\epsilon$ is of primary importance and that $R_{\lambda}$ is of secondary importance. However, this does not exclude the possibility that flow Reynolds number can play a role: (a) droplets respond to a range of turbulent eddies, including large-scale motion in low-Reynolds number DNS, (b) the limited scales in DNS do not really allow

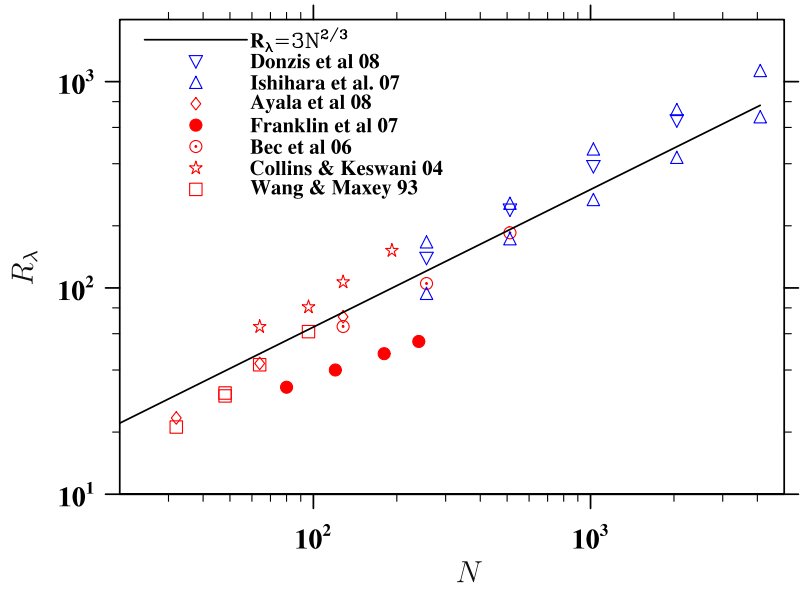

Fig. 1. Simulated flow Taylor microscale Reynolds number as a function of grid resolution. separation of effects of large and small scales, and (c) there exists a possibility that small-scale eddy structure is connected to largescale eddies through non-local eddy interactions. For these reasons, previous PPDNS results showed a significant dependence of particle-pair statistics on $R_{\lambda}$. The level of $R_{\lambda}$-dependence of radial relative velocity and RDF at high $R_{\lambda}$ range found in real clouds is not to be resolved by PPDNS and is currently unsettled (Collins and Keswani, 2004; Ayala et al., 2008b).

The limited scale range in PPDNS also makes it possible for the large-scale forcing scheme to affect the collision statistics of inertial particles. Both stochastic (Eswaran and Pope, 1988; Alvelius, 1999) and deterministic (Sullivan et al., 1994; Rosales and Meneveau, 2005) forcing schemes have been developed in single-phase turbulence simulations and both forms have been applied in PPDNS. Ayala et al. (2008a) speculated that some of the quantitative differences between different PPDNS studies could be due to the different forcing schemes used. No systematic study of the effects of different forcing schemes on collision statistics has been undertaken.

\subsection{Lagrangian tracking of particles}

Under the assumptions of dilute particulate loading and no particle-particle aerodynamic interaction, a general convenient form of the equation of motion for PPDNS is as follows:

$m_{p} \frac{d \mathbf{V}}{d t}=\left(m_{p}-m_{f}\right) \mathbf{g}+m_{f} \frac{D \mathbf{U}}{D t}+\frac{1}{2} m_{f}\left(\frac{d \mathbf{U}}{d t}-\frac{d \mathbf{V}}{d t}\right)+6 \pi a \mu(\mathbf{U}-\mathbf{V})$

where $\mu$ is fluid viscosity, $a$ is particle radius, $m_{p}=\frac{4}{3} \rho_{p} \pi a^{3}$, $m_{f}=\frac{4}{3} \rho_{f} \pi a^{3}$, the two Lagrangian derivatives are

$\frac{d}{d t}=\frac{\partial}{\partial t}+\mathbf{V} \cdot \nabla, \quad \frac{D}{D t}=\frac{\partial}{\partial t}+\mathbf{U} \cdot \nabla$

The above equation of motion includes particle inertia, body force, buoyancy force, fluid acceleration force, added mass, and Stokes drag. This is a simplified form of the general equation derived by Maxey and Riley (1983) after neglecting the Besset history term and Faxen corrections due to local curvature in the undisturbed fluid velocity field.

For Stokes disturbance flow, the two Lagrangian derivatives are close to one another (Maxey and Riley, 1983). Eq. (5) can then be written, in a compact form, as

$\frac{d \mathbf{V}}{d t}-\beta \frac{d \mathbf{U}}{d t}=(1-\beta) \mathbf{g}+\frac{\mathbf{U}-\mathbf{V}}{\tau_{p}}$,

where $\beta=3 \rho_{f} /\left(\rho_{f}+2 \rho_{p}\right)$, the inertial response time is $\tau_{p}=a^{2} /(3 v \beta)$, and $v=\mu / \rho_{f}$. The still-fluid terminal velocity is $\mathbf{W}=\tau_{p}(1-\beta) \mathbf{g}$.

The above equation of motion covers three limiting cases: (a) the heavy particle limit $\left(\rho_{p} \gg \rho_{f}\right.$ or $\left.\beta=0\right)$, (b) the microbubble limit $\left(\rho_{p} \ll \rho_{f}\right.$ or $\left.\beta=3\right)$, and (c) the fully buoyant particle $\left(\rho_{p}=\rho_{f}\right.$ or $\left.\beta=1\right)$. The microbubble limit was used in the PPDNS study of microbubbles in Wang and Maxey (1993b) and Maxey et al. (1997). For the fully buoyant particle limit, the equation of motion becomes solvable to yield $\mathbf{V}-\mathbf{U}=\left.(\mathbf{V}-\mathbf{U})\right|_{t=0} \exp \left(-t / \tau_{p}\right)$ with $\tau_{p}=a^{2} /(3 v)$ so the particle quickly approaches the fluid velocity. This implies that the above general form provides a consistent transition from light particle behavior to heavy particle behavior

Most studies of particle dynamics concern the heavy particle limit, with the following simplified equation of motion:

$\frac{d \mathbf{V}}{d t}=\mathbf{g}+f\left(\operatorname{Re}_{p}\right) \frac{\mathbf{U}-\mathbf{V}}{\tau_{p}}$ 
where $\tau_{p}=2 \rho_{p} a^{2} /(9 \mu)$. The correction factor $f\left(R e_{p}\right)$ is added to account for deviation from linear Stokes drag law, where $R e_{p}=2 a|\mathbf{V}-\mathbf{U}| / v$. A commonly used correlation is $f\left(R e_{p}\right)=$ $1+0.15 R e_{p}^{0.687}$ (Clift et al., 1978). Almost all PPDNS studies of turbulent collision performed to date consider this limit. The methodology reviewed in this paper could be easily applied to the more general equation of motion, Eq. (7).

Numerical integration of the equation of motion requires interpolation of fluid velocity (and acceleration if the general form is used) at the particle location from the values of the calculated fluid velocity at the grid points. Accurate interpolation is very important especially when turbulent collision is concerned. Several methods are available and are discussed in the literature (Yeung and Pope, 1988; Balachandar and Maxey, 1989; Rovelstad et al., 1994; Lekien and Marsden, 2005), including tri-linear, tri-cubic, cubic spline, Hermite, spectral, and high-order Lagrangian interpolations. The exact order needed depends on the resolution parameter $\left(k_{\max } \eta\right)$ used in the flow simulation, in general, we recommend a secondorder accuracy as a minimum. In our work, the highly accurate $6^{3}$-point Lagrangian interpolation has been used. The current trend of moving to MPI (Message Passing Interface) code implementation requires some reconsideration of the interpolation scheme in terms of balancing the accuracy with communication cost. Tricubic interpolation appears to be the optimal choice as it is more local in data storage (Lekien and Marsden, 2005; Homann et al., 2007).

The particles were introduced into the simulation when the background air turbulence had reached the statistically stationary stage. The initial conditions were that the locations of the droplets were randomly distributed and the initial velocity was set equal to the local fluid velocity plus the terminal velocity of the particle. After about $3 \times \max \left(\tau_{p 1}, \tau_{p 2}\right)$, data on collision-related statistics were begun to be accumulated to obtain running averages, to minimize any effect of the initial conditions.

\subsection{Collision detections and computation of kinematic properties}

To closely simulate the number density in clouds, typically on the order of $10^{5}-10^{6}$ droplets were followed. For turbulent collision involving this large number of droplets, the most computationally demanding task is the collision detection, a classical many-body interaction problem. The method for collision detection went through several iterations and the final version in our research utilized the efficient cell-index method and the concept of linked lists (Allen and Tildesley, 1987). A collision detection grid was carefully chosen so that all collision events were counted and, at the same time, no time was wasted on processing pairs with large separations.

An important issue is the post-collision treatment. The simplest case is so-called ghost particles where each particle continues to advance on its own after a collision or a geometric overlap with another particle, this ghost-particle treatment was shown by Wang et al. (1998a) to be consistent with the classical theoretical formulation of collision kernel by Saffman and Turner (1956). A more realistic treatment for cloud droplets is that, after two particles have collided, both of them are immediately removed and two new non-overlapping particles are randomly re-introduced in the computational domain (Zhou et al., 1998). Intermediate treatment between the two by simply excluding overlapping pairs at the beginning of a time step was also considered in Zhou et al. (1998). Perfect elastic collision treatment was used in Sundaram and Collins (1997) and Reade and Collins (1998), which could be appropriate for solid particles. The collision rate does, to some extent, depend on the exact post-collision treatment method used (Zhou et al., 1998).

Typically, a bi-disperse system of droplets of radius $a_{1}$ and $a_{2}$ was considered as a sub-system of a general polydisperse suspen- sion. While we were primarily interested in the 1-2 collision events, self collisions (1-1 and 2-2) were also detected. A separate code was used to independently compute the kinematic properties $\left\langle\left|w_{r}(r)\right|\right\rangle$ and $g_{12}(r)$ (Zhou et al., 2001). The separation distance between pairs was divided into narrow bins to compute the average kinematic statistics at a given separation. For further details on collision detections and computation of kinematic properties, the readers are referred to Zhou et al. (1998) and Zhou et al. (2001).

In summary, the study of geometric collision involves the following procedures at each time step:

(1) Interpolate the undisturbed fluid velocities at the locations of the droplets, $\mathbf{U}\left(\mathbf{Y}^{(k)}, t\right)$, using Lagrangian interpolation or other schemes.

(2) Advance the velocities and locations of the droplets using the equation of motion.

(3) Process collision detections and pair kinematic statistics.

(4) Advance the undisturbed fluid turbulence field $\mathbf{U}(\mathbf{x}, t)$ using a pseudo-spectral method.

\subsection{Major contributions}

Here we illustrate major contributions of PPDNS. While PPDNS has been used extensively to advance our understanding of single-particle transport (Riley and Patterson, 1974; Squires and Eaton, 1991b) and two-way coupling in particle-laden flows (Squires and Eaton, 1990; Elghobashi and Truesdell, 1993), here we limit our discussions primarily to turbulent collision. The early studies considered mostly inertial particles without sedimentation and were mainly directed toward two general questions: (1) how do air turbulence and preferential concentration affect the turbulent geometric collision rate? (2) how to represent the collision rate kinematically? The unique advantage of PPDNS is that both dynamic collision events and kinematic pair statistics can be quantified, a capability yet to be realized in experimental methods. The dynamic collision kernel is computed as $\Gamma_{12}^{\mathrm{D}}=\left\langle\dot{\mathscr{N}}_{12}\right\rangle /\left(n_{1} n_{2}\right)$, where $\left\langle\dot{\mathscr{N}}_{12}\right\rangle$ is the number of collision per unit time per unit volume and $n_{1}$ and $n_{2}$ are average number concentrations.

For monodisperse, non-settling inertial particles, the collision rate depends on the Stokes number, the ratio of $\tau_{p}$ to the Kolmogorov time $\tau_{k}$. Due to preferential concentration, the collision rate increases very quickly with $S t$ for $S t<1$, and peaks at a $\tau_{p}$ value between $\tau_{k}$ and the large-eddy time scale $T_{e}$ (Sundaram and Collins, 1997; Zhou et al., 1998). An example is shown in Fig. 2(a) by the curve marked monodisperse. This results from a combined effect of preferential concentration and turbulent transport, as the preferential concentration effect is most effective when $\tau_{p} \sim \tau_{k}$ (Fig. 2(b)), while the turbulent transport effect is maximized when $\tau_{p} \sim T_{e}$ (Fig. 2(c)).

The two effects can be separated by the following kinematic description of geometric collision kernel (Sundaram and Collins, 1997; Wang et al., 2000)

$\Gamma_{12}=2 \pi R^{2}\left\langle\left|w_{r}(r=R)\right|\right\rangle g_{12}(r=R)$,

where $w_{r}$ is the radial component of the relative velocity $\mathbf{w}$ between two particles, namely, $w_{r}=\mathbf{w} \cdot \mathbf{r} / r, \mathbf{r}$ is the relative separation vector, and $r=|\mathbf{r}|$. One important assumption is that the relative velocity $\mathbf{w}$ is incompressible, thus influx and outflux over the sphere surface are equal. The collision kernel for uniform particle distribution is then half the surface area multiplied by the average magnitude of the radial relative velocity. The enhancement factor $g_{12}(r)$ is the radial distribution function and measures the effect of preferential 
concentration on the pair number density at separation $r$. In direct numerical simulations, $g_{12}$ can be computed as, at any given time,

$g_{12}(r ; t)=\frac{N_{\text {pair }} / V_{s}}{N_{1} N_{2} / V_{\mathrm{B}}}$

where $N_{\text {pair }}$ is the total number of pairs detected with separation distance falling in a spherical shell of inner radius equal to $r_{1} \equiv r-\delta_{1}$ and outer radius equal to $r_{2} \equiv r+\delta_{2}$. Here $\delta_{1}$ and $\delta_{2}$ are small fractions of $r . V_{s}=4 \pi\left[\left(r+\delta_{2}\right)^{3}-\left(r-\delta_{1}\right)^{3}\right] / 3$ is the volume of the spherical shell. $N_{1}$ is the total number of size-1 particles used in the simulation, and $N_{2}$ is the total number of size-2 particles. $V_{B}$ is the volume of the computational domain. Both $w_{r}$ and $g_{12}$ are averaged over time and orientation.

The kinematic formulation extends the classical spherical formulation of Saffman and Turner (1956) and has been shown to be more general than the cylindrical formulation based on cylindrical interception volume (Wang et al., 1998b, 2005c). Using the dynamic collision kernel obtained directly from PPDNS, the kinematic formulation has been confirmed for inertial particles with and without sedimentation (Wang et al., 2000; Zhou et al., 2001; Ayala et al., 2008a). This is perhaps one of the most significant contributions made by PPDNS, providing a foundation for developing theoretical parameterization of turbulent collision kernel.

The two kinematic statistics have been carefully studied for both nonsettling (Sundaram and Collins, 1997; Wang et al., 2000; Zhou et al., 2001) and settling (Franklin et al., 2005, 2007; Ayala et al., 2008a) particles. Fig. 2 shows typical results from PPDNS, taken from Zhou et al. (2001), for nonsettling inertial particles. The essential message here is that both small-scale and large-scale turbulent motion affects the turbulent geometric collision kernel and kinematic pair statistics show rather complex dependence on the inertial response times in a bidisperse system. These PPDNS data have prompted a few theoretical studies (Zaichik and Alipchenkov, 2003; Zaichik et al., 2003, 2006; Derevich, 2007) where the data were used to validate theoretical development, in view of the current lack of equivalent experimental data.

Results of turbulent collision rate and pair statistics on sedimenting cloud droplets have been compiled in Franklin et al. (2007) and Ayala et al. (2008a). For cloud droplets, the level of enhancement of gravitational collision kernel by turbulence is of interest. Since sedimentation is always dominating the inertial effects (Wang et al., 2006) due to relatively small flow dissipation rates, the turbulent enhancement is fairly modest. The relative increase in geometric collision kernel by turbulence is typically below $50 \%$ at flow dissipation rate of $\epsilon=400 \mathrm{~cm}^{2} / \mathrm{s}^{3}$ (Franklin et al., 2007; Ayala et al., 2008a). Both perferential concentration and turbulent transport have been observed to play a role in this modest enhancement. Particle sedimentation was shown to decorrelate the concentration fields of two different particle sizes, making RDF much less than the level for monodisperse particles (Zhou et al., 2001; Wang et al., 2006; Ayala et al., 2008a). This modest enhancement, however, could have a noticeable impact on warm rain initiation (Xue et al., 2008).

In the above, we focus on pair statistics at contact. More generally, pair statistics at different separation distances have also been studied in PPDNS. For monodisperse particles of size less than the Kolmogorov length, RDF exhibits power-law scaling and the scaling exponent as a function of St has been studied through PPDNS (Reade and Collins, 2000; Falkovich and Pumir, 2004; Collins and Keswani, 2004).

Two related topics are the preferential concentration and the average settling velocity of inertial particles. The rigorous study by Maxey (1987) elucidated the physical origin of preferential concentration as a result of trajectory bias toward regions of low vor-

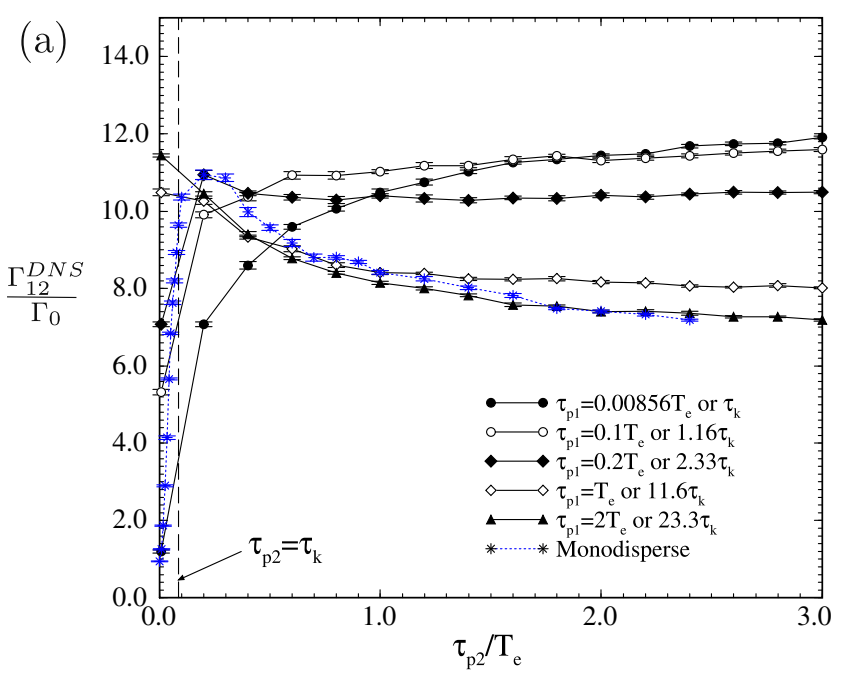

(b)
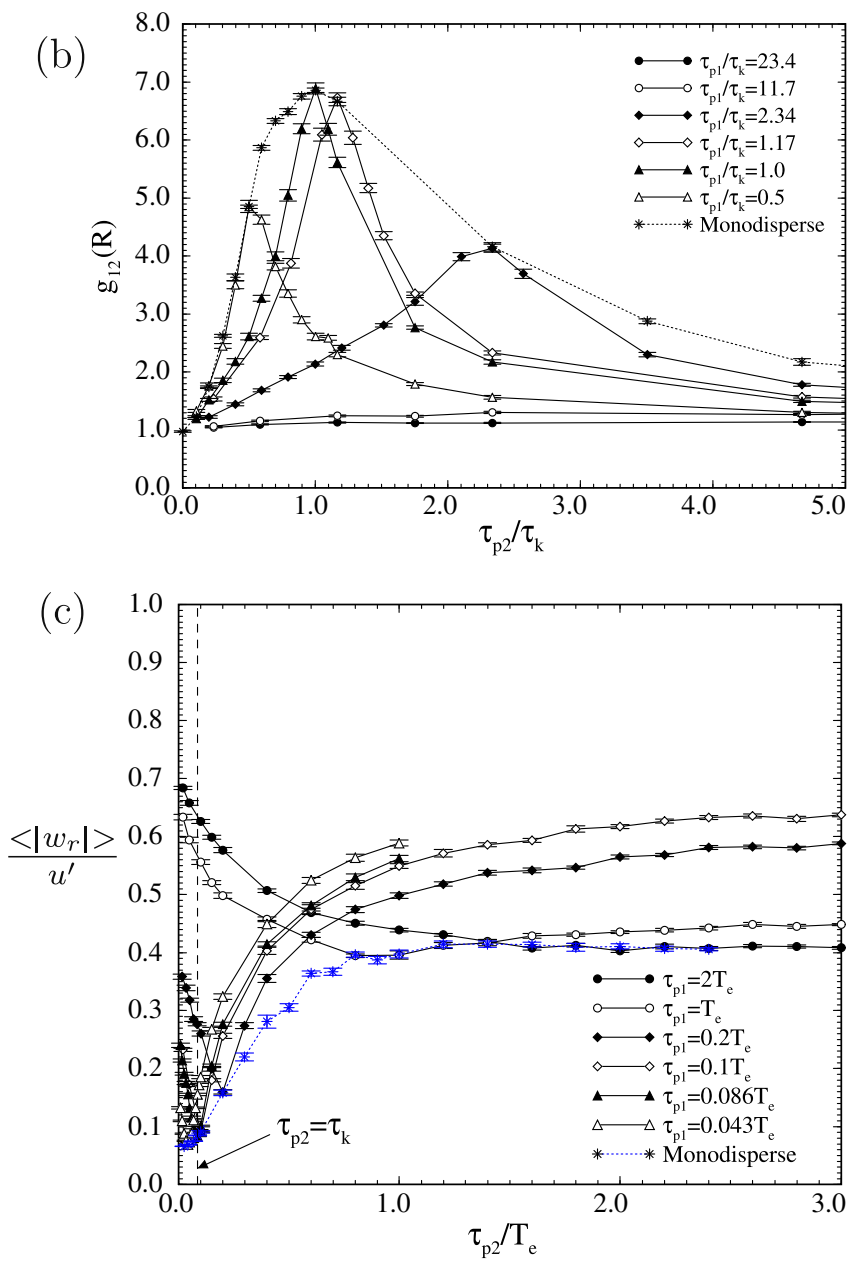

Fig. 2. Turbulent collision statistics taken from Zhou et al. (2001) at $R_{\lambda}=45$ : (a) Dynamic collision kernels, normalized by $\Gamma_{0}=(8 \pi / 15)^{1 / 2} R^{3} v_{k} / \eta$, as a function of $\tau_{p 2} / T_{e}$, (b) RDF as a function of $\tau_{p 2} / \tau_{k}$, and (c) the radial relative velocity $\left\langle\left|w_{r}\right|\right\rangle$, normalized by the fluid r.m.s. velocity $u^{\prime}$, as a function of particle inertia $\tau_{p 2} / T_{e}$.

ticity and high strain rate. PPDNS confirmed this striking phenomenon (Squires and Eaton, 1991a; Wang and Maxey, 1993) and showed that preferential concentration follows Kolmogorov scaling, namely, the maximum level of bias is realized when the particle inertial response time is comparable to $\tau_{k}$ (Wang and Maxey, 1993). The preferential concentration has since been extensively explored experimentally (Eaton and Fessler, 1994; Fallon 
and Rogers, 2002; Wood et al., 2005; Jaczewski and Malinowski, 2005) and theoretically (Elperin et al., 2002; Sigurgeirsson and Stuart, 2002; Zaichik and Alipchenkov, 2003; Chun et al., 2005; Duncan et al., 2005). The level of preferential concentration is significantly less for cloud droplets due to strong gravitational settling that reduces the effective interaction time of particles with a vortical structure. Fig. 3 shows a visualization taken from Ayala et al. (2008a).

A consequence of preferential concentration is a phenomenon known as preferential sweeping for sedimenting particles (Wang and Maxey, 1993), namely, sedimenting particles bias their trajectories toward regions of downward fluid motion around vortices and could settle significantly faster than the terminal velocity (Maxey, 1987; Wang and Maxey, 1993; Davila and Hunt, 2001). The increased settling occurs selectively for particles with a certain range of terminal velocity and inertial response time (Wang and Maxey, 1993; Davila and Hunt, 2001). The results of Wang and Maxey (1993) have been validated and extended in several experimental studies (Aliseda et al., 2002; Yang and Shy, 2005; Kawanisi and Shiozaki, 2008). Fig. 4 displays the ratio of average settling velocity of a droplet in turbulent air flow to its terminal velocity

(a)

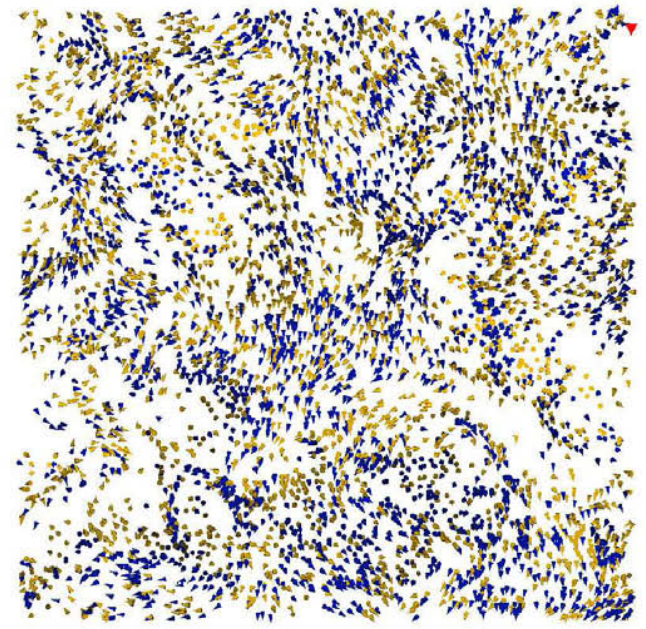

(b)

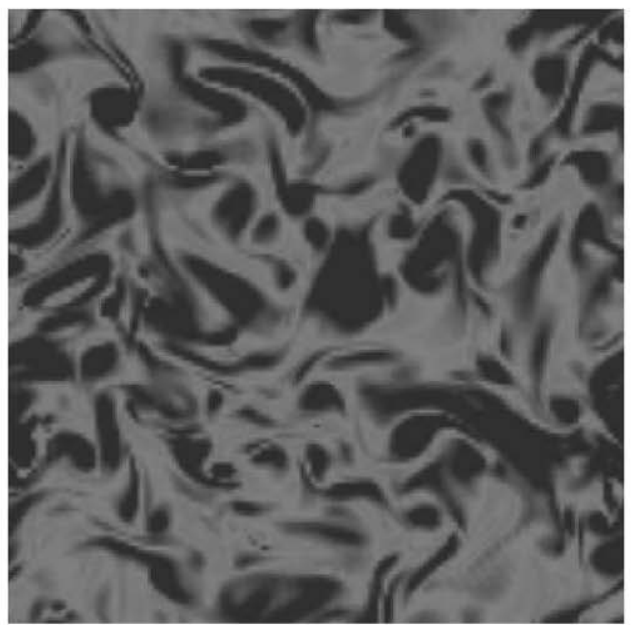

0.0

$$
0.5
$$

1.0

1.5

2.0

Fig. 3. A snapshot from DNS of droplet position (top) and normalized flow enstrophy (bottom) on a same planar slice. Top: the locations of droplets of $20 \mu \mathrm{m}$ radius are shown by yellow cones and those of $30 \mu \mathrm{m}$ by blue cones. The flow parameters are: $R_{\lambda}=72.41$ and $\epsilon=400 \mathrm{~cm}^{2} / \mathrm{s}^{3}$. Note that the $30 \mu \mathrm{m}$ droplets $(S t=0.570)$ show much higher levels of preferential concentration and $20 \mu \mathrm{m}$ droplets $(S t=0.253)$ display only a weak clustering.

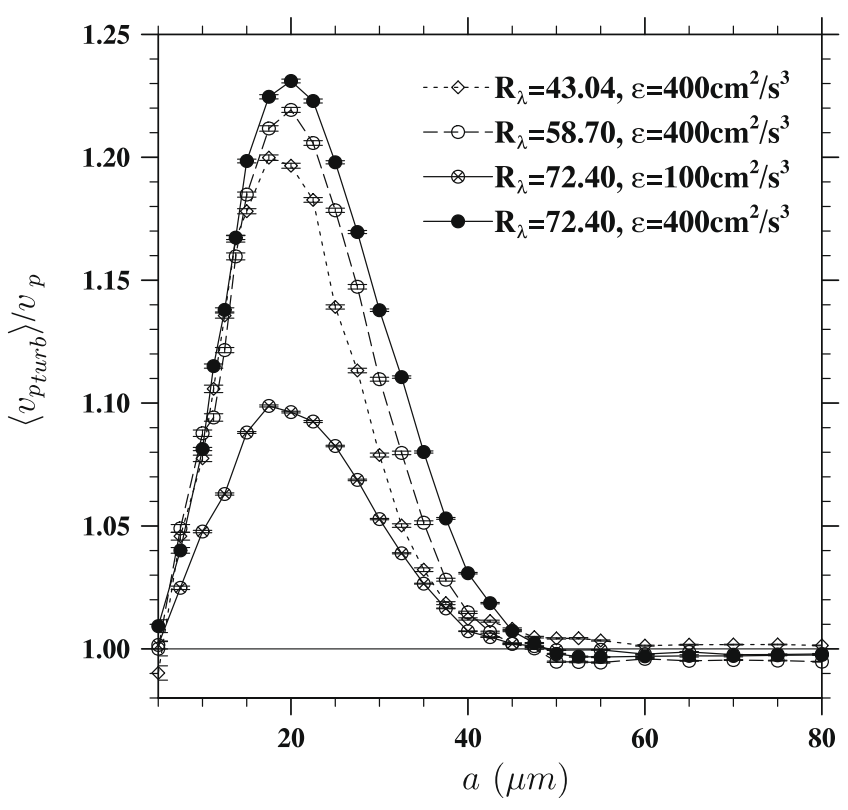

Fig. 4. The average settling velocity of droplets in a turbulent air, normalized by the terminal velocity in stagnant air.

in stagnant air. The PPDNS results show that large droplets $(a \geqslant 40 \mu \mathrm{m})$ move in a turbulent flow at a mean velocity roughly equal to the terminal velocity. Interestingly, $20-\mu \mathrm{m}$ cloud droplets always exhibit the largest increase in settling velocity. This can be explained by the theory of Davila and Hunt (2001) which showed that the increased settling in vortical flow is maximized when the parameter $F_{p}=\tau_{p}^{3} g^{2} / v$ is on the order of one (Davila and Hunt, 2001; Falkovich et al., 2002), which corresponds to roughly $20 \mu \mathrm{m}$ droplets. $F_{p}$ may be interpreted as the ratio of the inertial response time to the time $\left(v / v_{p}^{2}\right)$ for the droplet to settle through a Kolmogorov eddy.

In summary, PPDNS has been used productively to advance our understanding of turbulent collision, preferential concentration, and average settling rate of inertial particles.

\section{Hybrid DNS}

PPDNS does not address local droplet-droplet aerodynamic interaction and collision efficiency. Even in the absence of background air turbulence, the collision efficiency is a sensitive function of droplet sizes for droplets of radii less than $60 \mu \mathrm{m}$ (Klett and Davis, 1973; Pruppacher and Klett, 1997). It has been speculated for some time that air turbulence could significantly alter the collision efficiency (Almeida, 1976), but an accurate methodology to simulate this aspect has not been available.

Compared to the geometric collision, collision efficiency is a much more difficult problem as the disturbance flows introduce another set of length and time scales in addition to the background air turbulence. While there are quite a few studies in the literature concerning the collision efficiency of cloud droplets without air turbulence, there are very few studies devoted to the collision efficiency in a turbulent flow (Almeida, 1979; Grover and Pruppacher, 1985; Koziol and Leighton, 1996; Pinsky et al., 1999; Wang et al., 2005a; Pinsky et al., 2006). As pointed out in Wang et al. (2005a), these previous studies predicted different levels of enhancement on collision efficiency. This in part results from different kinematic formulations used to define the collision efficiency in different studies, some of which are not applicable to turbulent collisions. More importantly, there is currently a lack of 
accurate and consistent representations of aerodynamic interaction of many droplets in a turbulent flow.

\subsection{Methodology}

As a first step in developing a consistent computational method for treating aerodynamic interaction of cloud droplets in a turbulent flow, Wang et al. (2005b) introduced an improved superposition method (ISM) to study the collision efficiency of cloud droplets in still air. The basic idea is to impose, in some average sense, the no-slip boundary condition on the surface of each droplet to better determine the magnitude and coupling of the Stokes disturbance flows in a many-droplet system. The advantage of ISM is that the application to many-droplet interactions in a turbulent airflow is rather straightforward leading to a hybrid direct numerical simulation (HDNS) approach (Wang et al., 2005a; Ayala et al., 2007). The HDNS approach combines direct numerical simulation of the background air turbulence with an analytical representation of the disturbance flow introduced by many droplets. The approach takes advantage of the fact that the disturbance flow due to droplets is localized in space and there is a sufficient lengthscale separation between the droplet size and the Kolmogorov scale of the background turbulent flow. This hybrid approach provides, for the first time, a quantitative tool for studying the combined effects of air turbulence and aerodynamic interactions on the motion and collisional interactions of cloud droplets. The disturbance flow is coupled with the background air turbulence through the approximate implementation of the no-slip boundary conditions on each droplet. Dynamical features in three dimensions and on spatial scales ranging from a few tens of centimeters down to $10 \mu \mathrm{m}$ are captured. Both the near-field and the far-field droplet-droplet aerodynamic interactions could be incorporated (Wang et al., 2007).

In HDNS, the disturbance flows due to droplets are represented as localized Stokes flows. The flow field experienced by a droplet is then the combination of the undisturbed or background turbulent flow $\mathbf{U}(\mathbf{x}, t)$ and the disturbance flows of all other droplets. Since the Stokes disturbance flows are each governed by the linear Stokes equation, they can be superimposed to still satisfy the same Stokes equation locally (Wang et al., 2005b). This is the physical basis of the original superposition method (Langmuir, 1948; Pruppacher and Klett, 1997). The challenge is to satisfy the no-slip boundary conditions for all the particles in the system. Wang et al. (2005b) recognized that, by optimizing the magnitude of the disturbance flow experienced by each particle, it is possible to satisfy the no-slip boundary condition on the surface of each particle when averaged over the surface of the particle. Specifically, the fluid velocity of the composite flow at the center of each particle is equal to the velocity of that particle. This requirement leads to a more accurate representation of the force acting on a particle due to the disturbance flows by all other particles than the original superposition method.

In a turbulent carrier flow, the disturbance flow field in a system containing $N_{p}$ small particles can be written as

$\tilde{\mathbf{u}}(\mathbf{x}, t)=\sum_{k=1}^{N_{p}} \mathbf{u}_{S}\left(\mathbf{r}^{(k)} ; a^{(k)}, \mathbf{V}^{(k)}-\mathbf{U}\left(\mathbf{Y}^{(k)}, t\right)-\mathbf{u}^{(k)}\right)$,

where

$$
\begin{aligned}
\mathbf{u}_{S}\left(\mathbf{r}^{(k)} ; a^{(k)}, \mathbf{V}_{\mathbf{p}}^{(k)}\right) \equiv & {\left[\frac{3}{4} \frac{a^{(k)}}{r^{(k)}}-\frac{3}{4}\left(\frac{a^{(k)}}{r^{(k)}}\right)^{3}\right] \frac{\mathbf{r}^{(k)}}{\left(r^{(k)}\right)^{2}}\left(\mathbf{V}_{\mathbf{p}}^{(k)} \cdot \mathbf{r}^{(k)}\right) } \\
& +\left[\frac{3}{4} \frac{a^{(k)}}{r^{(k)}}+\frac{1}{4}\left(\frac{a^{(k)}}{r^{(k)}}\right)^{3}\right] \mathbf{V}_{\mathbf{p}}^{(k)}
\end{aligned}
$$

represents the Stokes disturbance flow due to the $k$ th droplet of radius $a^{(k)}$ moving at velocity $\mathbf{V}_{\mathbf{p}}^{(k)}$ in an otherwise quiescent fluid, and $\mathbf{r}^{(k)} \equiv \mathbf{X}-\mathbf{Y}^{(k)}$. Here $\mathbf{Y}^{(k)}$ is the instantaneous location of the $k$ th droplet. Eq. (12) is based on a single isolated particle and represents a combination of a Stokeslet and a potential dipole flow. In a multiparticle system the coefficients of each term could differ from those specified in Eq. (12). It may be desirable to include effects of local variations in the Stokes flow on the surface of the particle relative to the velocity at the center such as Faxen corrections. It would also be possible to include dipole terms (Kim and Karrila, 1991) in the above formulation to extend the capability of the approach.

Eq. (11) contains explicitly the disturbance flow velocity $\mathbf{u}^{(k)}$ at the location $\mathbf{Y}^{(k)}$ of the $k$ th droplet, due to all other droplets in the system. In Eq. (11), the combination $\left[\mathbf{V}^{(k)}-\mathbf{U}\left(\mathbf{Y}^{(k)}, t\right)-\mathbf{u}^{(k)}\right]$ represents the relative velocity between the $k$-th droplet and the composite flow $\widetilde{\mathbf{U}}(\mathbf{x}, t) \equiv \mathbf{U}(\mathbf{x}, t)+\tilde{\mathbf{u}}(\mathbf{x}, t)$ but excluding the disturbance flow due to the $k$ th droplet itself. Namely, $\mathbf{u}^{(k)}$ represents the disturbance flow velocity due to all droplets except the $k$ th droplet, at the location of the $k$ th droplet. $\mathbf{u}^{(k)}$ is determined by applying the center-point approximation (Wang et al., 2005b) to the boundary conditions $\widetilde{\mathbf{U}}\left(\left|\mathbf{r}^{(k)}\right|=a^{(k)}, t\right)=\mathbf{V}^{(k)}$, yielding

$$
\begin{aligned}
\mathbf{u}^{(k)} & =\underbrace{\sum_{m=1}^{N_{p}} \mathbf{u}_{s}\left(\mathbf{d}^{(m k)} ; \boldsymbol{a}^{(m)}, \mathbf{V}^{(m)}-\mathbf{U}\left(\mathbf{Y}^{(m)}, t\right)-\mathbf{u}^{(m)}\right), \text { for }}_{m \neq k} \\
k & =1,2, \ldots, N_{p},
\end{aligned}
$$

where $\mathbf{d}^{(m k)} \equiv \mathbf{Y}^{(k)}-\mathbf{Y}^{(m)}$. Therefore, $\mathbf{u}^{(k)}$ is a function of the background flow field and, the instantaneous locations and velocities of all particles. Eq. (13) implies that each disturbance flow velocity component at the location of the $k$ th particle will depend on all the disturbance flow velocity components of all other particles. Eq. (13) is a large linear system of dimension $3 N_{p}$.

Fig. 5 shows three grazing trajectories of $20-\mu \mathrm{m}$ droplets, relative to $25-\mu \mathrm{m}$ droplets. The locations of the $20-\mu \mathrm{m}$ droplets are shown at a fixed time interval. Clearly, as a $20-\mu \mathrm{m}$ droplet approached a $25-\mu \mathrm{m}$ droplet, the relative motion was slowed down. The relative trajectory of the $20-\mu \mathrm{m}$ droplet also bended around the $25-\mu \mathrm{m}$ droplet. Both resulted from the aerodynamic interaction. Unlike the still background air case, the grazing particles cover a much larger horizontal extent due to the background air turbulence.

The drag force acting on the $k$ th particle due to the interactions with the turbulent flow field and the disturbance flow field can be rigorously shown to be (Wang et al., 2005b)

$\mathbf{D}^{(k)}(t)=-6 \pi \mu a_{k}\left[\mathbf{V}^{(k)}(t)-\left(\mathbf{U}\left(\mathbf{Y}^{(k)}(t), t\right)+\mathbf{u}^{(k)}\right)\right]$.

Therefore, the equation of motion of any given particle " $k$ " is

$\frac{d \mathbf{V}^{(k)}(t)}{d t}=-\frac{\mathbf{V}^{(k)}(t)-\left(\mathbf{U}\left(\mathbf{Y}^{(k)}(t), t\right)+\mathbf{u}^{(k)}\right)}{\tau_{p}^{(k)}}-\mathbf{g}$

$\frac{d \mathbf{Y}^{(k)}(t)}{d t}=\mathbf{V}^{(k)}(t)$

where $\tau_{p}^{(k)}=2 \rho_{p}\left(a^{(k)}\right)^{2} /(9 \mu)$ is the particle inertial response time.

In this HDNS approach, the disturbance flows will incorporate naturally the droplet-droplet hydrodynamic interactions when droplets are in close proximity on the scale of droplet diameter. During each time step, the following procedures are performed:

(1) Advance the undisturbed fluid turbulence field $\mathbf{U}(\mathbf{x}, t)$ using a pseudo-spectral method. 


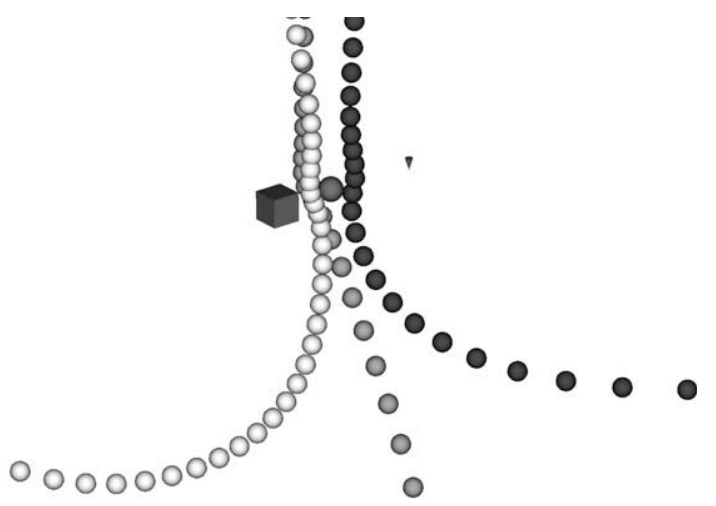

Fig. 5. Three grazing trajectories of $20-\mu \mathrm{m}$ droplets relative to $25-\mu \mathrm{m}$ droplets with hydrodynamic interactions in a turbulent suspension at $\epsilon=400 \mathrm{~cm}^{2} / \mathrm{s}^{3}$. The time interval was set to $0.0022 \mathrm{~s}$ or about $42 \%$ the inertial response time of the $20-\mu \mathrm{m}$ droplet. The small cube has an edge length equal to $10 \%$ flow Kolmogorov length.

(2) Interpolate the undisturbed fluid velocities at the locations of the droplets, $\mathbf{U}\left(\mathbf{Y}^{(k)}, t\right)$.

(3) Solve the disturbance flow velocity $\mathbf{u}^{(k)}$ experienced by each droplet, using Eq. (13).

(4) Advance the velocities and locations of the droplets.

(5) Detect droplet-droplet collision events and calculate relevant kinematic and dynamic properties.

The determination of $\mathbf{u}^{(k)}$ amounts to solving a linear system of dimension $3 N_{p}$. The Gauss-Seidel method is used to solve the system iteratively as described in detail in Ayala et al. (2007). A truncation radius was assumed to limit the range of the hydrodynamic interaction. It was shown that the collision efficiency is not sensitive to this truncation radius (Ayala et al., 2007), although singleparticle statistics depend strongly on the truncation radius (Wang et al., 2007).

\subsection{Results and outlook}

In HDNS, particles were not allowed to overlap in space. Using HDNS, Wang et al. (2005a) demonstrated that the kinematic formulation, Eq. (9), is still valid if corrections due to this non-overlap requirement are taken. This is an important realization and allows a consistent definition of turbulent collision efficiency. The overall enhancement factor $\eta_{T}$, the ratio of turbulent collision kernel to the hydrodynamic gravitation kernel, is then a product of the enhancement of the geometric collision, $\eta_{G}$, and the enhancement of the collision efficiency, $\eta_{E}$ (Wang et al., 2005a).

We found that the net enhancement factor $\eta_{T}=\eta_{E} \eta_{G}$ is typically in the range of one to five (Wang et al., 2008). It tends to be larger when the droplets are rather different in size or of nearly equal in size (Fig. 6). In the first limiting case, the gravitational hydrodynamic kernel is small due to small differential sedimentation. In the second limiting case, the gravitational hydrodynamic kernel may also be small due to small collision efficiency. Therefore, air turbulence plays an important role in enhancing the collision kernel in both limiting cases. The values of $\eta_{E}$ and $\eta_{G}$ from our HDNS have been tabulated in Ayala et al. (2008a) and Wang et al. (2008). The level of increase in the collision efficiency for cross-size collisions depends primarily on the flow dissipation rate and the size ratio $a_{2} / a_{1}$. For instance, the collision efficiency between droplets of $18 \mu \mathrm{m}$ and $20 \mu \mathrm{m}$ in radii is increased, relative to the gravitational collision efficiency in stagnant air, by a factor of 4 and 1.6 by air turbulence at dissipation rates of $400 \mathrm{~cm}^{2} / \mathrm{s}^{3}$ and $100 \mathrm{~cm}^{2} / \mathrm{s}^{3}$, respectively. For most cross-size collisions, aerodynamic interactions reduce the average radial relative velocity but also increase the radial distribution function. The collision efficiency for self-collisions in a bidisperse turbulent suspension can be larger than one. Such an increase in self-collisions is related to the far-field manybody aerodynamic interaction and may depend on the volumetric concentration of droplets of all sizes in the system.

HDNS provides a general framework for a systematic improvement of the approach. In this regard, the HDNS approach is closely related to the multipole expansion method of Durlofsky et al. (1987), also in general known as the Stokesian dynamics approach (Brady and Bossis, 1988). In fact, the center-point formulation of ISM is essentially the zero-moment expansion with only monopole terms and without Faxen correction, while the integral formulation of ISM is the zero-moment expansion with the Faxen correction since the integral average of disturbance flow velocity over a droplet surface is equivalent to the center-point velocity plus the Faxen term. Durlofsky et al. (1987) presented a multipole formulation known as the Force-Torque-Stresslet (FTS) formulation which includes moments up to the first-order plus Faxen terms. This multipole expansion method considers many-body interaction with Stokes disturbance flows superimposed onto a nonuniform background flow.

The HDNS approach outlined above cannot handle correctly short-range or lubrication forces (Wang et al., 2005b). As a logical next step, we have recently developed an efficient approach for treating the hydrodynamic interaction of two spherical particles settling under gravity (Rosa et al., submitted for publication). Droplets are assumed to be small such that the fluid inertia in the disturbance flows may be neglected. An effort is made to ensure accuracy of the method for any inter-particle separation by considering three separation ranges. The first is the long-range interaction where a multipole method is applied. Explicit formulae for drag force and torque are derived from the FTS formulation of Durlofsky et al. (1987). The FTS formulation is found to be accurate when the separation distance normalized by the average radius is larger than 5 for all cases. The second range concerns the shortrange interaction where the interaction force could be very large.

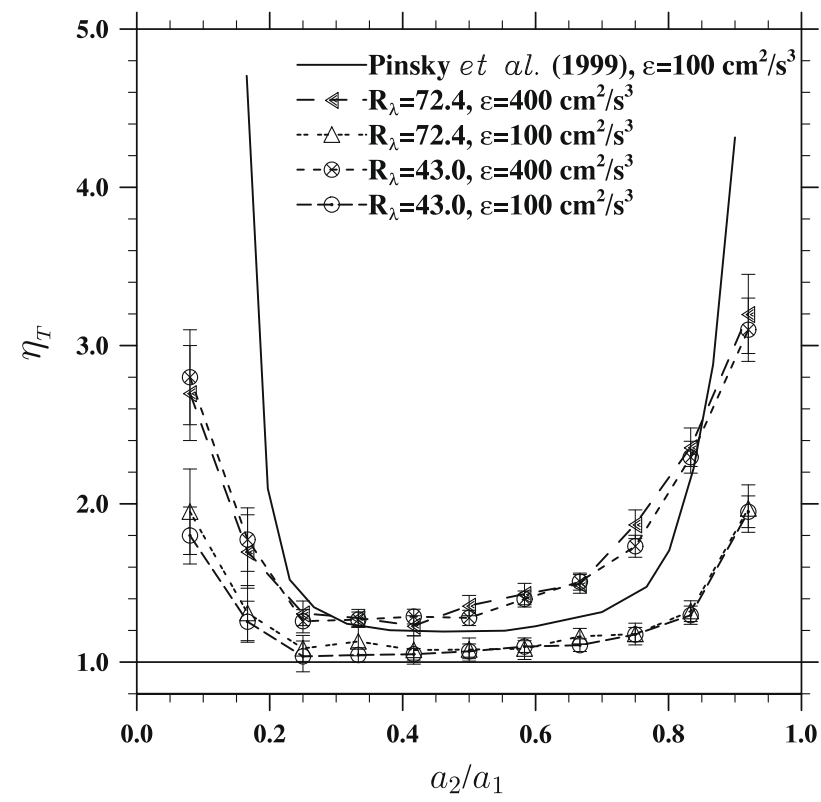

Fig. 6. The net enhancement factor, the ratio of the turbulent collection kernel and the hydrodynamic-gravitational collection kernel, as a function of $a_{2} / a_{1}$ for $a_{1}=30 \mu \mathrm{m}$. The largest enhancements occur at small size ratio or for nearly equal-size pairs, in qualitative (but not quantitative) agreement with the results in Pinsky et al. (1999). In the legend, $\epsilon$ is the flow viscous dissipation rate and $R_{\lambda}$ is the Taylor microscale Reynolds number of the simulated background turbulent air flow. 
The leading-order lubrication expansions of Jeffrey and Onishi (1984) are employed for this range and are found to be accurate when the normalized separation is less than about 0.01 for all cases. Finally, for the intermediate range where no simple method is available, a third-order polynomial fitting is proposed to bridge the long-range and short-range interactions. After optimizing the exact form of polynomial fitting and the boundary locations for the three separation ranges, the force representation is found to be highly accurate when compared with the exact solution for Stokes flows. Using this efficient method, collision efficiencies for the case of gravitational interaction have been calculated. It is shown that the results of collision efficiency are in excellent agreement with previous results based on more complex treatment of the interaction force (Fig. 7). In Fig. 7, an attractive van der Waals force of the form given in Davis (1984) was added when the equation of motion was solved. The van der Waals force applies to two droplets along the direction of their separation vector and is significant only when two droplets are very close to one another. When the van der Waals force overcomes the Stokes aerodynamic lubrication force, two droplets can come into contact leading to coalescence. It is then no longer necessary to assume a small cut-off separation for geometric contact that would otherwise be necessary should the van der Waals force not be included explicitly. It is hoped that this method can be applied to treat hydrodynamic interactions of many particles in either stagnant or turbulent background flow.

\section{Point-particle based LES}

For many industrial applications, a simulation must address the exterior boundaries of a flow system. It is not feasible to resolve all scales of fluid turbulence. Unlike the bottom-up approach of fluid turbulence in PPDNS or HDNS, a more practical approach would be to apply large-eddy simulation (LES), a top-down computational approach. LES is relatively mature for single-phase turbulent flows, but not so for turbulent flows laden with inertial particles due to the lack of a reliable subgrid scale (SGS) model (Marchioli et al., 2008).

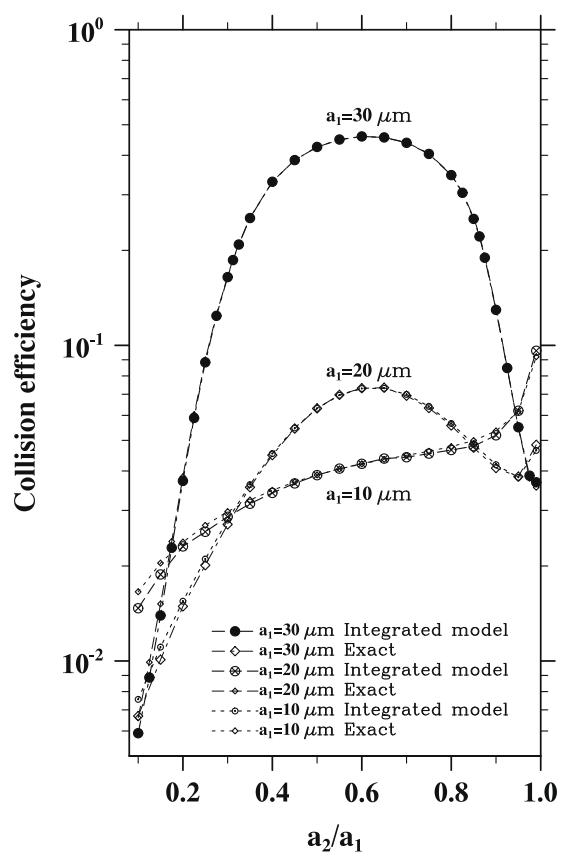

In LES, large-scale motions are explicitly calculated while the effect of small-scale motions on the resolved flow field is represented by the SGS model. The application of LES to inertial particles raises some new challenges. The first is the SGS models for velocity fields. Most of the currently-existing SGS models are based on energy budget equations and could thus predict the spatial statistics, such as energy spectra, but they may not ensure the correct prediction of temporal statistics such as Lagrangian velocity correlations (He et al., 2002). The latter is very important to the LES prediction of single-particle and particle-pair dispersion in turbulent flows. Therefore, it is necessary to develop a SGS model which could also ensure accurate prediction of the temporal statistics. The second aspect concerns the SGS models for particle motions. A particle trajectory in LES is calculated from the resolved part of fluid velocity field. Namely, the unresolved part of fluid velocity field is missing in the LES context. If the initial separation of particle pair is less than the grid scale, the absence of subgrid fluid motion leads to under-estimation of the pair relative motion and thus the rate of pair dispersion (Yang et al., 2008). The third challenge is the lack of subgrid vortical structures and their related time evolution which may govern particle dynamics such as preferential concentration - a demixing phenomenon strongly controlled by subgrid flow structures. If the particle inertial response time is smaller than the characteristic time of resolved eddies, weaker particle clustering will result in LES (Goto and Vassilicos, 2006; Marchioli et al., 2008). These issues are relevant to turbulent collision of inertial particles.

Since Lagrangian characteristics of fluid motion are of fundamental importance to particle-laden turbulence, as the first step it is necessary to study the effects of SGS modeling on the motion of fluid particles (Wang et al., 1995; Wang and Squires, 1996; Armenio et al., 1999). A systematic study of Lagrangian statistics of fluid particles by Yang et al. (2008) concludes that a LES with an eddy viscosity SGS model could correctly predict one-particle statistics but may not predict correctly two-particle statistics. Similar trends are expected for inertial particles. Armenio et al. (1999) pointed out that inertial particles are less sensitive to the SGS fluctuations than the fluid particles. Therefore, the LES prediction of single-particle statistics could be less sensitive to the subgrid fluid

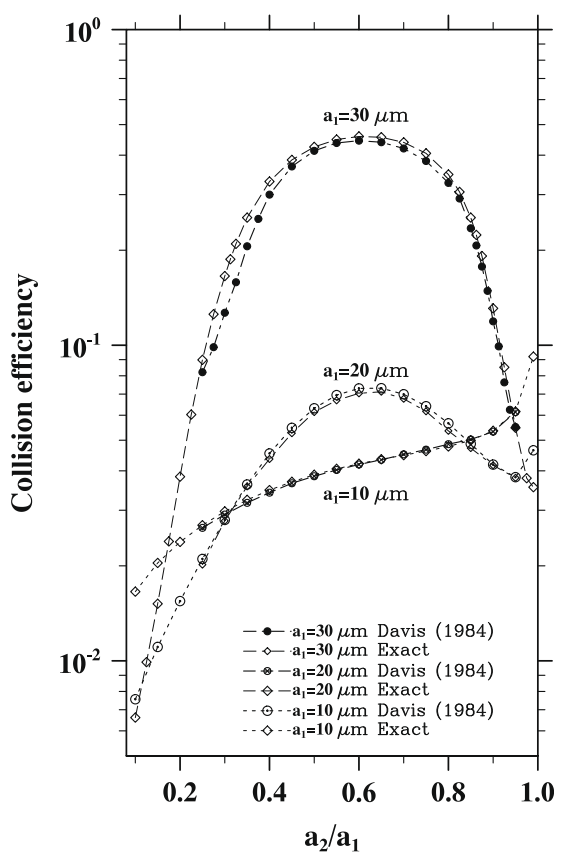

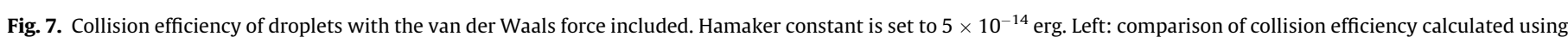

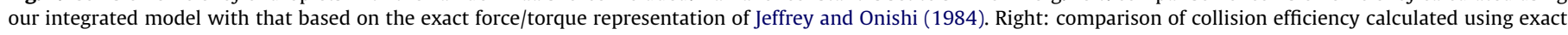
force/torque representation of Jeffrey and Onishi (1984) and the results of Davis (1984). 
motion. However, the inertial particles have some additional complexity, such as enhanced settling velocity and preferential concentration. Yeh and Lei (1991) and Yang and Lei (1998) compared the Lagrangian statistics of LES with DNS for inertial particles and quantified the contribution of SGS motions to the settling velocity of heavy particles. Shotorban and Mashayek (2005) and Kuerten (2006) developed a deconvolution approach to compute the Lagrangian statistics of inertial particles in turbulent channel flows. The deconvolution approach can partially recover the unsolved velocity fields. Recently, Kuerten and Verman (2005) and Shotorban and Mashayek (2006) have developed a stochastic Lagrangian particle model to better represent Lagrangian statistics of inertial particles. Fede and Simonin (2006) found that the particle accumulation and collision rate are significantly influenced by the SGS velocity fluctuations when the particle response time is of the same order or smaller than the subgrid Lagrangian integral time scale measured along particle path.

We carried out point-particle based LES of both fluid particles and inertial particles and compared results with those obtained

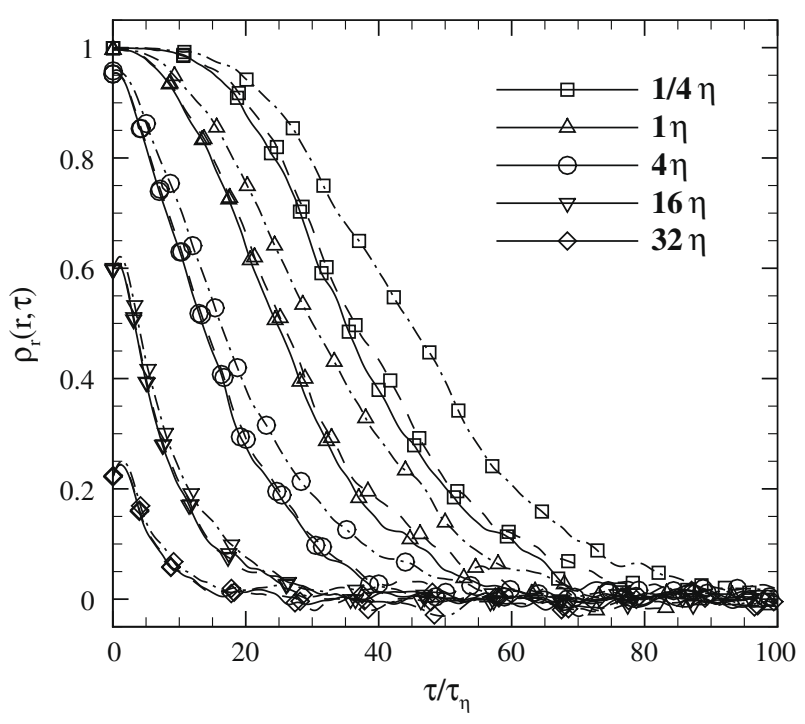

Fig. 8. The normalized Lagrangian velocity correlation of fluid particle pairs of different initial separations: Solid lines for DNS $128^{3}$, dashed lines for LES $64^{3}$ and dash-dotted lines for LES $32^{3}$. The symbols correspond to the different initial separations and $\eta$ denotes the Kolmogorov length. from PPDNS. The DNS flow was at $128^{3}$ resolution with $R e_{\lambda}=80$, while two grid resolutions of $64^{3}$ and $32^{3}$ were used in LES with the spectral eddy viscosity SGS model. Here results on pair dispersion and collision rate are discussed. Fig. 8 plots the temporal evolution of the normalized Lagrangian velocity correlation between two fluid particles initially separated at a distance of $r$. The LES results decays more slowly than those from DNS. The slower decay is evidently more noticeable for the smaller initial separations than that for larger initial separations. A possible explanation for the slower decay is that the Lagrangian velocity field in LES is more correlated than that in DNS. The exclusion of small scale motions in LES makes the velocity fields less intermittent. Meanwhile, the eddy viscosity SGS model plays a role of dissipation which reduces the fluctuations of LES velocity fields more intensively than the molecular dissipation alone. Both these factors yield an over-prediction of Lagrangian two-particle velocity correlations.

Fig. 9 shows the collision rate of monodisperse heavy particles versus the Stokes number using the data from DNS, filtered DNS and LES. Non-sedimenting heavy particles were considered. The collision rate is proportional to the collision kernel as the number concentration was kept constant. A total of 32,000 particles were followed. The three sets of results show the same trends qualitatively: the collision rates increase very rapidly for small Stokes numbers with a peak at the Stokes number $S t=4.0$ and then drop slowly with increasing Stokes number. This is consistent with the results of Sundaram and Collins (1997) and Zhou et al. (2001). The filtered DNS collision rate is very similar to DNS collision rate for all particle Stokes numbers, despite about 32\% of enstrophy is removed in the filtered DNS field. However, the LES collision rate is $10 \%$ smaller than the DNS collision rate, although the cut-off wavenumber in LES is the same as that in the filtered DNS. This apparently was due to the altered energy spectrum near the cutoff wavenumber in LES, as compared to DNS. As a result, the realized fluid kinetic energy in LES was about $10 \%$ smaller than that of DNS flow.

In summary, the general observation is that single-particle Lagrangian statistics can be well predicted by LES, but particle-pair statistics such as pair velocity correlations and collision rate may not be correctly represented in LES. Both the effect of subgrid scales on resolved scales and the missing subgrid scales themselves affect two-particle statistics. This suggests the need of adding a SGS model for particle dynamics in addition to the conventional SGS fluid velocity model. Much work is needed in this direction to develop and validate SGS model for the particulate phase, as well as comparison with DNS simulations at higher flow Reynolds numbers.

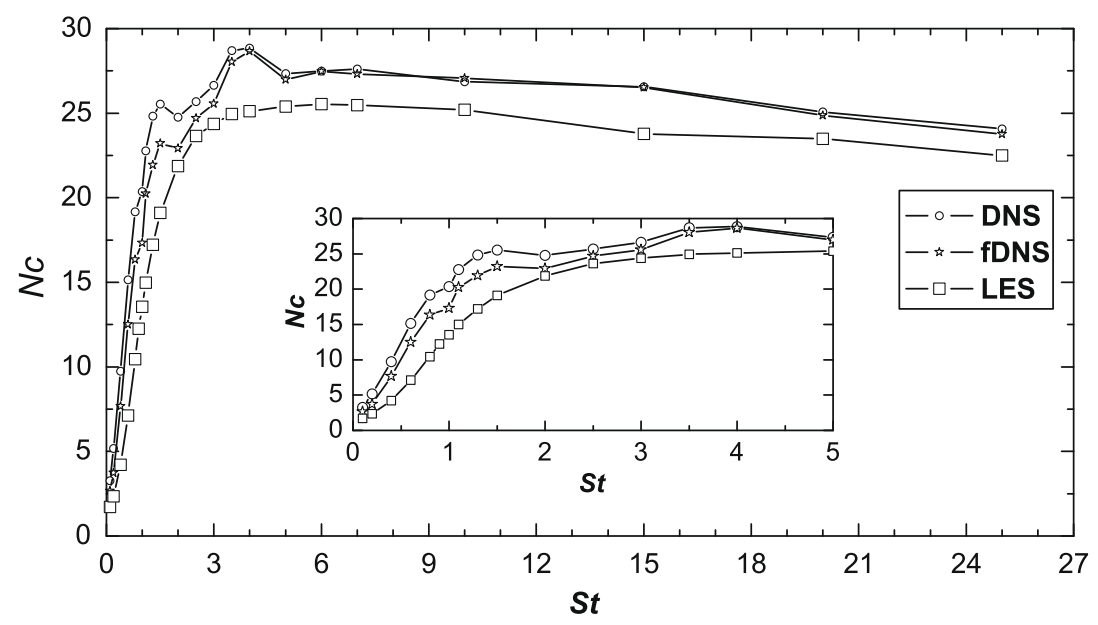

Fig. 9. The collision rates of inertial particles versus the Stokes numbers obtained from DNS at $128^{3}$, filtered DNS with cutoff wavenunmber $k_{c}=10$ and LES at $32^{3}$. 
In spite of these SGS modeling issues in LES, we do believe that LES presents a better alternative for numerical prediction of particle-laden turbulence when compared to Reynolds averaged Navier-Stokes approach as the spatial structure and dynamics of large-scale turbulent motion are explicitly simulated in LES.

\section{Particle-resolved DNS}

One limitation of the treatment above is the assumption of Stokes disturbance flows, which is known to become inaccurate for droplets larger than $30 \mu \mathrm{m}$ in radii (Klett and Davis, 1973). On the one hand, currently, no known method can treat, in an efficient manner, the problem of many-droplet interactions beyond Stokes disturbance flows. The work of Klett and Davis (1973) represents the first study in which the leading-order fluid-inertia (or finite droplet Reynolds number) effect in the disturbance flows is considered for two-droplet interaction, by using Oseen flow equations. Several attempts (Shafrir and GalChen, 1971; Lin and Lee, 1975; Schlamp et al., 1976; Pinsky et al., 2001) were made to handle two-droplet aerodynamic interaction at finite Reynolds numbers using a simple superposition method in which the disturbance flow due to each droplet is computed numerically by solving nonlinear Navier-Stokes equations, without any influence by the disturbance flow due to the other droplet. Unfortunately, such a simple superposition method has been widely criticized as it can result in unphysical collision efficiency (Schlamp et al., 1976), and it is known to be very inaccurate even for Stokes disturbance flows (Wang et al., 2005b). It is not surprising that no attempt has been made to adopt this simple superposition method to many-droplet interactions.

In recent years, several groups have made efforts to develop particle-resolved simulation methods, with the main goal to better understand flow modulation by particles. These include the Stokesian-flow approach of Pan and Banerjee (1996), the immersed boundary method of Uhlmann (2005), the control volume approach of Sharma and Patankar (2005), the force coupling method of Maxey and Patel (2001) and Dance and Maxey (2003), and the semi-analytical method of Prosperetti and co-workers (Takagi et al., 2003, 2005). Other methods such as the body-fitted finiteelement method and the Lattice Boltzmann method may also have the potential to be used for turbulent disperse flow with finite-size particles, but so far they have mainly been used for suspension flows without carrier-fluid turbulence. A noticeable exception is the study of particle collision in a liquid-solid turbulent flow using the Lattice Boltzmann method by Ten Cate et al. (2004).

Recently, we started to develop particle-resolved flow simulation tool (Gao and Wang, 2007), using the computational approach (i.e. Physalis) developed by Prosperetti and co-workers (Zhang and Prosperetti, 2003, 2005). Our ultimate goal is to address collisionefficiency at finite droplet Reynolds number involving many-body interaction. The basic idea of Physalis is to recognize that, because of the no-slip boundary conditions on its surface, a droplet induces a specific local flow structure that could be used to linearize the Navier-Stokes equations in the neighborhood of the droplet surface. The fluid velocity, pressure, and vorticity near the droplet surface can be expressed analytically using series solutions of Stokes flow equations (Zhang and Prosperetti, 2003, 2005; Gao and Wang, 2007). As a result, the geometric surface of the droplet can be replaced by a Stokes flow solution valid in a narrow but finite region near the surface, known as the cage region. An important advantage of this hybrid method is that the force and torque acting on the particle can be calculated directly from the expansion coefficients of the Stokes solution, avoiding integration from the numerical solution.
Fig. 10 shows a two-dimensional simulation of particle-particle aerodynamic interaction. The well-known scenario of drafting, kissing, and tumbling has been reproduced. Similar results have been obtained in three dimensions. We are currently working to extract
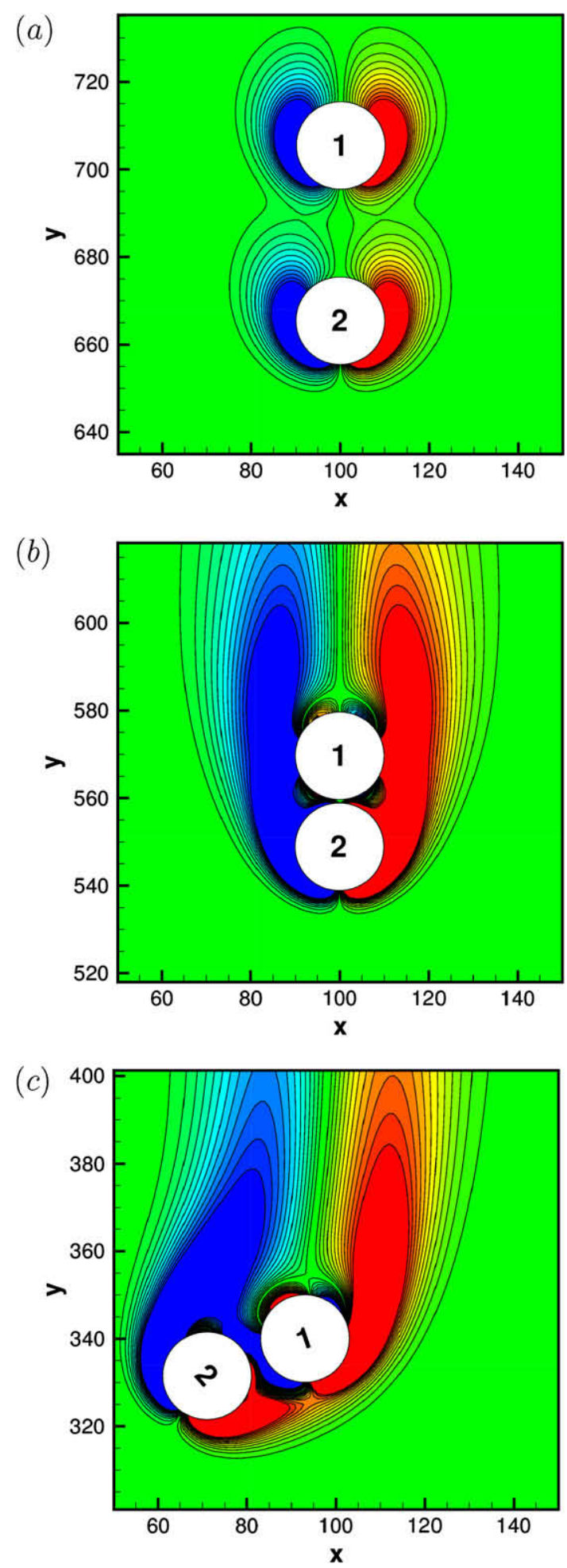

Fig. 10. A two-dimensional size-resolved simulation of particle-particle aerodynamic interaction in a confined channel of width equal to 20 particle radius, demonstrating the sequence of (a) drafting, (b) kissing, and (c) tumbling. The Reynolds number based on maximum particle sedimentation velocity was around 35 . The color contours show the flow vorticity distribution. The numbers inside the particles indicate their relative location and rotation. (For interpretation of the references to color in this figure legend, the reader is referred to the web version of this paper.) 
forces of different physical origins acting on the particles from these particle-resolved simulations.

A careful examination of all particle-resolved simulation methods reveals a difficulty in resolving short-range interaction force that is essential for studying collision efficiency. This difficulty is not unexpected as particle-resolved simulations are not designed to resolve directly short-range interaction and flow detail. A short-range force-interaction model has to be introduced and Dance et al. (2004) showed that results could be sensitive to this modeling. We are currently exploring the possibility of embedding an analytical representation of two-body near-field lubrication flow, such as the method of Sangani and Mo (1994) and Mo and Sangani (1994), into the hybrid particle-resolved approach.

\section{Summary and concluding remarks}

We have provided an overview of point-particle based and hybrid simulations developed to better understand and quantify turbulent collision of inertial particles, a multi-scale and threedimensional dynamic problem of particle-laden turbulent flow. Turbulent collision of inertial particles involves an array of topics in fundamental fluid mechanics. PPDNS and HDNS have their unique advantages as well as limitations which have been delineated based on our own experience. Currently, advances in turbulent collision of inertial particles are mainly made through these simulation tools. The results from these simulations have validated and extend the kinematic formulation of the turbulent collision kernel.

Even at its simplest level, PPDNS provides fundamental information on all relevant dynamic and kinematic pair statistics. The current challenge is to push PPDNS to higher resolution and higher flow Reynolds numbers. The recent PPDNS study of inertial particles by Bec et al. (2006) combined $512^{3}$ flow simulation at $R_{\lambda}=185$ with Lagrangian tracking of up to 120 million particles. Indeed, since the disturbance flow due to particles is modelled by the equation of motion, PPDNS can treat a very large number of particles. Further advances in PPDNS require efficient MPI implementation of fluid turbulence, velocity interpolation, particle tracking, and collision detection, along with perhaps petascale computing resources. The MPI implementation issues related to the particulate phase are not trivial and interdisciplinary collaborations may be required.

The study of collision efficiency due to particle-particle hydrodynamic interactions in a turbulent background flow requires hybrid approaches beyond PPDNS. Our recent work on HDNS illustrates the possibility of including hydrodynamic interaction, although much remains to be done to improve the accuracy of short-range interaction.

There is a multi-dimensional parameter space (Stokes number, nondimensional sedimentation, density ratio, flow Reynolds number, dissipation rate, etc.) for turbulent collision of particles covering many natural processes and industrial applications. Only a portion of this parameter space has been explored, therefore, PPDNS and HDNS will continue to be used in various contexts to address different parameter combinations.

The radial distribution function accounts for collision enhancement due to preferential concentration. Very little is known theoretically how to model RDF for finite-inertia sedimenting particles at different flow Reynolds numbers (Ayala et al., 2008b). Most theoretical developments to date consider weak-inertia non-sedimenting particles. A comprehensive review of the theoretical modeling of RDF and turbulent collision kernel can be found in Ayala et al. (2008b). Likewise, experimental measurements of turbulent collision have not yet reached to a point where direct comparisons with PPDNS and HDNS can be made with confidence for all pair separation distances. Parallel advances in theory and exper- iment are essential in view of the Reynolds-number limitation of PPDNS and HDNS.

Large-eddy simulations and particle-resolved simulations could also be explored to address issues that could not be addressed by PPDNS and HDNS, such as effects of large-scale background turbulence, hydrodynamic interaction at finite particle Reynolds numbers, and effect of droplet deformation. It is desirable that all different computational tools are combined to extend the capability of any single approach.

Finally, as computational tools become more sophisticated, education of students could be challenging. We have attempted to illustrate essential outstanding issues and pertinent literature so students working on turbulent collision and other related topics would find this paper to be useful.

\section{Acknowledgments}

This study has been supported by the National Science Foundation (ATM-0114100, ATM-0527140 and ATM-0730766), the National Center for Atmospheric Research (NCAR), Chinese Academy of Sciences under the Innovative Project "Multi-scale modeling and simulation in complex systems" (KJCX-SW-L08), National Basic Research Program of China (973 Program) under Project No. 2007CB814800, and National Natural Science Foundation of China under Project Nos. 10325211, 10628206 and 10732090.

\section{References}

Abrahamson, J., 1975. Collision rates of small particles in a vigorously turbulent fluid. Chem. Eng. Sci. 30, 1371-1379.

Aliseda, A., Cartellier, A., Hainaux, F., Lasheras, J.C., 2002. Effect of preferential concentration on the settling velocity of heavy particles in homogeneous isotropic turbulence. J. Fluid Mech. 468, 77-105.

Allen, M.P., Tildesley, D.J., 1987. Computer Simulation of Liquids. Oxford University Press, Oxford

Almeida, F.C.de, 1976. The collisional problem of cloud droplets moving in a turbulent environment. Part I: a method of solution. J. Atmos. Sci. 33, 15711578.

Almeida, F.C.de, 1979. The collisional problem of cloud droplets moving in a turbulent environment. Part II: turbulent collision effiencies. J. Atmos. Sci. 36, 1564-1576.

Alvelius, K., 1999. Random forcing of three-dimensional homogeneous turbulence. Phys. Fluids 11, 1880-1889.

Armenio, V., Piomelli, U., Fiorotto, V., 1999. Effect of subgrid scales on particle motion. Phys. Fluids 11, 3030-3042.

Ayala, O., Grabowski, W.W., Wang, L.-P., 2007. A hybrid approach for simulating turbulent collisions of hydrodynamically-interacting particles. J. Comput. Phys. 225, 51-73.

Ayala, O., Rosa, B., Wang, L.-P., Grabowski, W.W., 2008a. Effects of turbulence on the geometric collision rate of sedimenting droplets: part 1 . Results from direct numerical simulation. New J. Phys. 10, 075015

Ayala, A., Rosa, B., Wang, L.-P., 2008b. Effects of turbulence on the geometric collision rate of sedimenting droplets: Part 2. Theory and parameterization. New J. Physics 10, 075016.

Balachandar, S., Maxey, M.R., 1989. Methods for evaluating fluid velocities in spectral simulations of turbulence. J. Comput. Phys. 83, 96-125.

Bec, J., Biferale, L., Bofftta, G., Celani, A., Cencini, M., Lanotte, A., Musacchio, S., Toschi, F., 2006. Acceleration statistics of heavy particles in turbulence. J. Fluid Mech. 550, 349-358.

Brady, J.F., Bossis, G., 1988. Stokesian dynamics. Annu. Rev. Fluid Mech. 20, 111157.

Chun, J., Koch, D., Rani, S.L., Ahluwalia, A., Collins, L.R., 2005. Clustering of aerosol particles in isotropic turbulence. J. Fluid Mech. 536, 219-251.

Clift, R., Grace, J.R., Weber, M.E., 1978. Bubbles Drops and Particles. Academic Press, New York.

Collins, L.R., Keswani, A., 2004. Reynolds number scaling of particle clustering in turbulent aerosols. New J. Phys. 6. Article No. 119.

Dance, S.L., Maxey, M.R., 2003. Incorporation of lubrication effcts into the forcecoupling method for particulate two-phase flow. J. Comput. Phys. 189, 212-238.

Dance, S.L., Climent, E., Maxey, M.R., 2004. Collision barrier effects on the bulk flow in a random suspension. Phys. Fluids 16, 828-831.

Davila, J., Hunt, J.C.R., 2001. Settling of small particles near vortices and in turbulence. J. Fluid Mech. 440, 117-145.

Davis, R.H., 1984. The rate of coagulation of a dilute polydisperse system of sedimenting spheres. J. Fluid Mech. 145, 179-199.

Delichatsios, M.A., 1980. Particle coagulation in steady turbulent flows: application to smoke aging. J. Colloid Interface Sci. 78, 163-174. 
Derevich, I.V., 2007. Coagulation kernel of particles in a turbulent gas flow. I. Int. J. Heat Mass Transfer 50, 1368-1387.

Donzis, D.A., Yeung, P.K., Sreenivasan, K.R., 2008. Dissipation and enstrophy in isotropic turbulence: resolution effects and scaling in direct numerical simulations. Phys. Fluids 20, 045108.

Duncan, K., Mehlig, B., Ostlund, S., Wilkinson, M., 2005. Clustering by mixing flows. Phys. Rev. Lett. 95. Article No. 240602.

Durlofsky, L., Brady, J.F., Bossis, G., 1987. Dynamic simulation of hydrodynamically interacting particles. J. Fluid Mech. 180, 21-49.

Duru, P., Koch, D.L., Cohen, C., 2007. Experimental study of turbulence-induced coalescence in aerosols. Int. J. Multiphase Flow 33, 987-1005.

Eaton, J.K., Fessler, J.R., 1994. Preferential concentration of particles by turbulence. Int. J. Multiphase Flow 20, 169-209.

Elghobashi, Truesdell, 1993. On the 2-way interaction between homogeneous turbulence and dispersed solid particles. 1. Turbulence modifiation. Phys. Fluids A $5,1790-1801$.

Elperin, T., Kleeorin, N., L'vov, V.S., Rogachevskii, I., Sokoloff, D., 2002. Clustering instability of the spatial distribution of inertial particles in turbulent flows. Phys. Rev. E 66. Article No. 036302.

Eswaran, E., Pope, S.B., 1988. An examination of forcing in direct numerical simulations of turbulence. Comput. Fluids 16, 257-278.

Falkovich, G., Pumir, A., 2004. Intermittent distribution of heavy particles in a turbulent flow. Phys. Fluids 16, L47-L50.

Falkovich, G., Fouxon, A., Stepanov, M.G., 2002. Acceleration of rain initiation by cloud turbulence. Nature 419, 151-154.

Fallon, T., Rogers, C.B., 2002. Turbulence-induced preferential concentration of solid particles in microgravity conditions. Exp. Fluids 33, 233-241.

Fede, P., Simonin, O., 2006. Numerical study of the subgrid fluid turbulence effects on the statistics of heavy colliding particles. Phys. Fluids, Article No. 045103.

Flagan, R.C., Seinfeld, J.H., 1988. Fundamentals of Air Pollution Engineering. Prentice-Hall, Englewood Cliff, NJ.

Franklin, C.N., Vaillancourt, P.A., Yau, M.K., Bartello, P., 2005. Collision rates of cloud droplets in turbulent flow. J. Atmos. Sci. 62, 2451-2466.

Franklin, C.N., Vaillancourt, P.A., Yau, M.K., 2007. Statistics and parameterizations of the effect of turbulence on the geometric collision kernel of cloud droplets. J. Atmos. Sci. 64, 938-954.

Gao, H., Wang, L.-P., 2007. Paper 713, CD-ROM Proceedings of the Sixth International Conference on Multiphase Flow, Leipzig, Germany, July 9-17, 2007.

Goto, S., Vassilicos, J.C., 2006. Slef similar clustering of inertial particles and zeroacceleration points in fully developed two-dimensional turbulence. Phys. Fluids $18,115103$.

Grover, S.N., Pruppacher, H.R., 1985. The effect of vertical turbulent fluctuations in the atmosphere on the collection of aerosol-particles by cloud drops. J. Atmos. Sci. 42, 2305-2318.

He, G.-W., Rubinstein, R., Wang, L.-P., 2002. Effects of subgrid-scale modeling on time correlations in large eddy simulation. Phys. Fluids 14, 2186-2193.

Homann, H., Dreher, J., Grauer, R., 2007. Impact of the floating-point precision and interpolation scheme on the results of DNS of turbulence by pseudo-spectral codes. Comput. Phys. Commun. 177, 560-565

Ishihara, T., Kaneda, Y., Yokokawa, M., Itakura, K., Uno, A., 2007. Small-scale statistics in high-resolution direct numerical simulation of turbulence: Reynolds number dependence of one-point velocity gradient statistics. J. Fluid Mech. 592, 335-366.

Jaczewski, A., Malinowski, S.P., 2005. Spatial distribution of cloud droplets in a turbulent cloud-chamber flow. Quarterly J. Roy. Meteor. Soc. 131, 2047-2062.

Jeffrey, D.J., Onishi, Y., 1984. Calculation of the xr resistance and mobility functions for two unequal rigid spheres in low-Reynolds-number flow. J. Fluid Mech. 139, 261-290.

Kawanisi, K., Shiozaki, R., 2008. Turbulent effcts on the settling velocity of suspended sediment. J. Hydraul. Eng.-ASCE 134, 261-266.

Kim, S., Karrila, S.J., 1991. Microhydrodynamics: Principles and Selected Applications, Butterworth-Heinemann, Boston, 1991, 507pp.

Klett, J.D., Davis, M.H., 1973. Theoretical collision efficiencies of cloud droplets at small Reynolds numbers. J. Atmos. Sci. 30, 107-117.

Koch, D.L., Hill, R.J., 2001. Inertial effects in suspension and porous-media flows. Annu. Rev. Fluid Mech. 33, 619-647.

Koziol, A.S., Leighton, H.G., 1996. The effect of turbulence on the collision rates of small cloud drops. J. Atmos. Sci. 53, 1910-1920.

Kuerten, J.G.M., 2006. Subgrid modeling in particle-laden channel flow. Phys. Fluids 18,025108

Kuerten, J.G.M., Verman, A.W., 2005. Can turbuphoresis be predicted by large-eddy simulation? Phys. Fluids 17, 011701.

Langmuir, I., 1948. The production of rain by a chain reaction in cumulus clouds at temperatures above freezing. J. Meteorol. 5, 175-192.

Lekien, F., Marsden, J., 2005. Tricubic interpolation in three dimensions. Int. J. Numer. Method Eng. 63, 455-471.

Lewis, D.M., Pedley, T.J., 2000. Planktonic contact rates in homogeneous isotropic turbulence: theoretical predictions and kinematic simulations. J. Theor. Biol. 205, 377-408.

Lin, C., Lee, S., 1975. Collision effiency of water drops in the atmosphere. J. Atmos. Sci. 32, 1412-1418.

Marchioli, C., Salvetti, M.V., Soldati, A., 2008. Some issues concerning large-eddy simulation of inertial particle dispersion in turbulent bounded flows. Phys. Fluids 20, 040603

Maxey, M.R., 1987. The gravitational settling of aerosol-particles in homogeneous turbulence and random flow fields. J. Fluid Mech. 174, 441-465.
Maxey, M.R., Patel, B.K., 2001. Localized force representation for particles sedimenting in Stokes flow. Int. J. Multiphase Flow 27, 1603-1626.

Maxey, M.R., Riley, J.J., 1983. Equation of motion for a small rigid sphere in a nonuniform flow. Phys. Fluids 26, 883-889.

Maxey, M.R., Patel, B.K., Chang, E.J., Wang, L.-P., 1997. Simulations of dispersed turbulent multiphase flow. Fluid Dyn. Res. 20, 143-156.

Mo, S.B., Sangani, A.S., 1994. A method for computing Stokes-flow interactions among spherical objects and its application to suspensions of drops and porous particles. Phys. Fluids 6, 1637-1652.

O'Rourke, P.J., Bracco, F.V., 1980. Modelling of drop interactions in thick sprays and a comparison with experiments. In: Stratified Charge Automotive Engines Conference, The Institution of Mechanical Engineers, London, p. 101.

Pan, Y., Banerjee, S., 1996. Numerical simulation of particle interactions with wall turbulence. Phys. Fluids 8, 2733-2755.

Pinsky, M.B., Khain, A.P., 2004. Collisions of small drops in a turbulent flow. Part II: effects of flow accelerations. J. Atmos. Sci. 61, 1926-1939.

Pinsky, M.B., Khain, A.P., Shapiro, M., 1999. Collisions of small drops in a turbulent flow. Part I: collision efficiency. Problem formulation and preliminary results. J. Atmos. Sci. 56, 2585-2600.

Pinsky, M.B., Khain, A.P., Shapiro, M., 2001. Collision efficiency of drops in a wide range of Reynolds numbers: effects of pressure on spectrum evolution. J. Atmos Sci. 58, 742-764.

Pinsky, M.B., Khain, A.P., Grits, B., Shapiro, M., 2006. Collisions of small drops in a turbulent flow. Part III: Relative droplet fluxes and swept volumes. J. Atmos. Sci. 63, 2123-2139.

Pinsky, M.B., Khain, A.P., Shapiro, M., 2007. Collisions of small drops in a turbulent flow. Part IV: droplet hydrodynamic interaction. J. Atmos. Sci. 64, 2462-2482.

Pruppacher, H.R., Klett, J.D., 1997. Microphysics of Clouds and Precipitation. Kluwer Academic Publishers, Dordrecht. 954pp.

Reade, W.C., Collins, L.R., 1998. Collision and coagulation in the infinite Stokesnumber regime. Aerosol Sci. Technol. 29, 493-509.

Reade, W.C., Collins, L.R., 2000. Effct of preferential concentration on turbulent collision rates. Phys. Fluids 12, 2530-2540.

Riley, J.J., Patterson, G.S., 1974. Diffusion experiments with numerically integrated isotropic turbulence. Phys. Fluids 17, 292-297.

Rosa, B., Wang, L.-P., Maxey, M.R., Grabowski, W.W. submitted for publication. An accurate model for aerodynamic interactions of cloud droplets, J. Atmos. Sci.

Rosales, C., Meneveau, C., 2005. Linear forcing in numerical simulations of isotropic turbulence: Physical space implementations and convergence properties. Phys Fluids 17, 095106.

Rovelstad, A.L., Handler, R.A., Bernard, P.S., 1994. The effct of interpolation errors on the Lagrangian analysis of simulated turbulent channel flow. J. Comput. Phys. 110, 190-195.

Saffman, P.G., Turner, J.S., 1956. On the collision of drops in turbulent clouds. J. Fluid Mech. 1, 16-30

Salazar, J.P.L.C., De Jong, J., Cao, L.J., Woodward, S.H., Meng, H., Collins, L.R., 2008 Experimental and numerical investigation of inertial particle clustering in isotropic turbulence. J. Fluid Mech. 600, 245-256.

Sangani, A.S., Mo, S.B., 1994. Inclusion of lubrication forces in dynamic simulations Phys. Fluids 6, 1653-1662.

Schlamp, R.J., Grover, S.N., Pruppacher, H.R., Hamielec, A.E., 1976. J. Atmos. Sci. 33 1747-1755.

Schmitt, F.G., Seuront, L., 2008. Intermittent turbulence and copepod dynamics: increase in encounter rates through preferential concentration. J. Marine Syst. 70, 263-272.

Shafrir, U., Gal-Chen, T.A., 1971. Numerical study of collision effiencies and coalescence parameters for droplet pairs with radii up to 300 microns. J. Atmos. Sci. 28, 741-751.

Sharma, N., Patankar, N.A., 2005. A fast computation technique for the direct numerical simulation of rigid particulate flows. J. Comput. Phys. 205, 439-457.

Shaw, R.A., 2003. Particle-turbulence interactions in atmospheric clouds. Annu. Rev. Fluid Mech. 35, 183-227.

Shotorban, B., Mashayek, F., 2005. Modeling subgrid-scale effects on particles by approximate deconvolution. Phys. Fluids 17, 081701.

Shotorban, B., Mashayek, F., 2006. A stochastic model for particle motion in largeeddy simulation. J. Turbul. 7, 1-13.

Sigurgeirsson, H., Stuart, A.M., 2002. A model for preferential concentration. Phys Fluids 14, 4352-4361.

Squires, K.D., Eaton, J.K., 1990. Particle response and turbulence modification in isotropic turbulence. Phys. Fluids A 2, 1191-1203.

Squires, K.D., Eaton, J.K., 1991a. Preferential concentration of particles by turbulence. Phys. Fluids A 3, 1169-1179.

Squires, K.D., Eaton, J.K., 1991b. Measurements of particle dispersion obtained from direct numerical simulations of isotropic turbulence. J. Fluid Mech. 226, 1-35.

Sullivan, N.P., Mahalingam, S., Kerr, R.M., 1994. Deterministic forcing of homogeneous, isotropic turbulence. Phys. Fluids 6, 1612-1614.

Sundaram, S., Collins, L.R., 1997. Collision statistics in an isotropic, particle-laden turbulent suspension. J. Fluid Mech. 335, 75-109.

Takagi, S., Oguz, H.N., Zhang, Z., Prosperetti, A., 2003. PHYSALIS: a new method for particle simulation. Part II: two-dimensional Navier-Stokes flow around cylinders. J. Comput. Phys. 187, 371-390.

Ten Cate, A., Derksen, J.J., Portela, L.M., Van Den Akker, H.E.A., 2004. Fully resolved simulations of colliding monodisperse spheres in forced isotropic turbulence. J. Fluid Mech. 519, 233-271.

Uhlmann, M., 2005. An immersed boundary method with direct forcing for the simulation of particulate flows. J. Comput. Phys. 209, 448-476. 
Wang, L.-P., Maxey, M.R., 1993. Settling velocity and concentration distribution of heavy particles in homogeneous isotropic turbulence. J. Fluid Mech. 256, 27-68.

Wang, L.-P., Maxey, M.R., 1993b. The motion of microbubbles in a forced isotropic and homogeneous turbulence. Appl. Sci. Res. 51, 291-296.

Wang, Q., Squires, K.D., 1996. Large eddy simulation of particle-laden turbulent channel flow. Phys. Fluids 8, 1207-1223.

Wang, Q., Squires, K.D., Wu, X., 1995. Lagrangian statistics in turbulent channel flow. Atmos. Env. 29, 2417-2427.

Wang, L.-P., Wexler, A.S., Zhou, Y., 1998a. On the collision rate of small particles in isotropic turbulence. Part 1. Zero-inertia case. Phys. Fluids 10, 266-276.

Wang, L.-P., Wexler, A.S., Zhou, Y., 1998b. Statistical mechanical descriptions of turbulent coagulation. Phys. Fluids 10, 2647-2651.

Wang, L.-P., Wexler, A.S., Zhou, Y., 2000. Statistical mechanical descriptions of turbulent coagulation of inertial particles. J. Fluid Mech. 415, 117-153.

Wang, L.-P., Ayala, O., Kasprzak, S.E., Grabowski, W.W., 2005a. Theoretical formulation of collision rate and collision efficiency of hydrodynamicallyinteracting cloud droplets in turbulent atmosphere. J. Atmos. Sci. 62, 2433-2450.

Wang, L.-P., Ayala, O., Grabowski, W.W., 2005b. Improved formulations of the superposition method. J. Atmos. Sci. 62, 1255-1266.

Wang, L.-P., Ayala, O., Xue, Y. 2005c. Reconciling the cylindrical formulation with the spherical formulation in the kinematic descriptions of collision kernel. Phys. Fluids 17, Article No. 067103.

Wang, L.-P., Ayala, O., Xue, Y., Grabowski, W.W., 2006. Comments on droplets to drops by turbulent coagulation. J. Atmos. Sci. 63, 2397-2401.

Wang, L.-P., Ayala, O., Grabowski, W.W., 2007. Effects of aerodynamic interactions on the motion of heavy particles in a bidisperse suspension. J. Turbul. 8.1, 1-28. doi:10.1080/14685240701233426.

Wang, L.-P., Ayala, O., Rosa, B., Grabowski, W.W., 2008. Turbulent collision efficiency of cloud droplets. New J. Phys. 10, 075013.

Williams, J.J.E., Crane, R.I., 1979. Drop coagulation in cross-over pipe flows of wet steam. J. Mech. Eng. Sci. 21, 357-360.

Wood, A.M., Hwang, W., Eaton, J.K., 2005. Preferential concentration of particles in homogeneous and isotropic turbulence. Int. J. Multiphase Flow 31, 1220-1230.
Xiong, Y., Pratsinis, S., 1991. Gas phase production of particles in reactive turbulent flows. J. Aerosol. Sci. 22, 637-655.

Xue, Y., Wang, L.-P., Grabowski, W.W., 2008. Growth of cloud droplets by turbulent collision-coalescence. J. Atmos. Sci. 65, 331-356.

Yang, C.Y., Lei, U., 1998. The role of the turbulent scales in the settling velocity of heavy particles in homogeneous isotropic turbulence. J. Fluid Mech. 371, 179205.

Yang, T.S., Shy, S.S., 2005. Two-way interaction between solid particles and homogeneous air turbulence: particle settling rate and turbulence modification measurements. J. Fluid Mech. 526, 171-216.

Yang, Y., He, G.-W., Wang, L.-P., 2008. Effects of subgrid scale modeling on Lagrangian statistics in large-eddy simulation. J. Turbul. 9, 1-24.

Yeh, F., Lei, U., 1991. On the motion of small particles in a homogeneous isotropic turbulent flow. Phys. Fluids A 3, 2571-2586.

Yeung, P.K., Pope, S.B., 1988. An algorithm for tracking fluid particles in numerical simulations of homogeneous turbulence. J. Comput. Phys. 79, 373-416.

Zaichik, L.I., Alipchenkov, V.M., 2003. Pair dispersion and preferential concentration of particles in isotropic turbulence. Phys. Fluids 15, 1776-1787.

Zaichik, L.I., Simonin, O., Alipchenkov, V.M., 2003. Two statistical models for predicting collision rates of inertial particles in homogeneous isotropic turbulence. Phys. Fluids 15, 2995-3005.

Zaichik, L.I., Simonin, O., Alipchenkov, V.M., 2006. Collision rates of bidisperse inertial particles in isotropic turbulence. Phys. Fluids 18, Article Number 035110.

Zhang, Z., Prosperetti, A., 2003. A method for particle simulation. J. Appl. Mech. Trans. ASME 70, 64-74.

Zhang, Z., Prosperetti, A., 2005. A second-order method for three-dimensional particle simulation. J. Comput. Phys. 210, 292-324.

Zhou, Y., Wexler, A.S., Wang, L.-P., 1998. On the collision rate of small particles in isotropic turbulence. Part 2. Finite-inertia case. Phys. Fluids 10 1206-1216.

Zhou, Y., Wexler, A.S., Wang, L.-P., 2001. Modelling turbulent collision of bidisperse inertial particles. J. Fluid Mech. 433, 77-104. 